\title{
JSAP1/JIP3 and JLP regulate kinesin-1-dependent axonal transport to prevent neuronal degeneration
}

\author{
T Sato ${ }^{1}$, M Ishikawa ${ }^{1}$, M Mochizuki ${ }^{1}$, M Ohta $^{1}$, M Ohkura ${ }^{2}$, N Nakai ${ }^{2}$, N Takamatsu ${ }^{3}$ and K Yoshioka ${ }^{*, 1}$
}

Axonal transport is critical for neuronal development and function, and defective axonal transport has been implicated in neurodegenerative diseases. However, how axonal transport is regulated, or how defective transport leads to neuronal degeneration, remains unclear. Here, we report that c-Jun $\mathrm{NH}_{2}$-terminal kinase (JNK)/stress-activated protein kinase-associated protein 1 (JSAP1, also known as JNK-interacting protein 3 (JIP3)) and JNK-associated leucine zipper protein (JLP) are essential for postnatal brain development. Mice with a double-knockout (dKO) in Jsap1 and JIp in the dorsal telencephalon developed progressive neuron loss. Using a primary neuron culture system with induced disruption of targeted genes, combined with gene rescue experiments, we show that JSAP1 and JLP regulate kinesin-1-dependent axonal transport with functional redundancy. We also show that the binding of JSAP1 and JLP to kinesin-1 heavy chain is crucial for interactions between kinesin-1 and microtubules. Furthermore, we describe a molecular mechanism by which defective kinesin-1-dependent axonal transport in Jsap1:Jlp dKO neurons causes axonal degeneration and subsequent neuronal death. JNK hyperactivation because of increased intra-axonal $\mathrm{Ca}^{2+}$ in the Jsap1:Jlp dKO neurons was found to mediate both the axonal degeneration and neuronal death, in cooperation with the $\mathrm{Ca}^{2+}$-dependent protease calpain. Our results indicate that axonal JNK may relocate to the nucleus in a dynein-dependent manner, where it activates the transcription factor c-Jun, resulting in neuronal death. Taken together, our data establish JSAP1 and JLP as positive regulators of kinesin-1-dependent axonal transport, which prevents neuronal degeneration. Cell Death and Differentiation (2015) 22, 1260-1274; doi:10.1038/cdd.2014.207; published online 9 January 2015

Axonal transport is essential for neuronal development and function, and disrupted axonal transport is an important pathophysiological factor in a variety of neurodegenerative diseases. ${ }^{1,2}$ However, the molecular mechanisms that regulate axonal transport, and how disruption of that transport leads to neuronal degeneration, are poorly understood. Long-distance transport in axons is driven by the kinesin and dynein motor proteins, which carry cargoes along microtubule (MT) tracks. MTs are uniformly oriented in the axon, with their plus ends toward the axon terminal and their minus ends toward the cell body. Kinesin-1 is a plus enddirected anterograde motor composed of two kinesin heavy chains (KHCs) and two kinesin light chains (KLCs). ${ }^{3}$ Neuronal kinesin-1 often transports its cargoes through adaptor proteins such as c-Jun $\mathrm{NH}_{2}$-terminal kinase (JNK)-interacting protein 1 (JIP1) and JNK/stress-activated protein kinase-associated protein 1 (JSAP1, also known as JIP3 or Sunday Driver). ${ }^{4}$

The JIP protein family includes JIP $1,{ }^{5}$ JIP2, ${ }^{6}$ JSAP1/JIP3 (Ito et al. 1999; Kelkar et al. 2000) and JNK-associated leucine zipper protein (JLP). ${ }^{9}$ Splice variants of JLP are known as JIP4
(Kelkar et al. 2005) or SPAG9. ${ }^{11}$ JIP proteins were first identified as scaffold proteins for specific JNK and p38 mitogen-activated protein kinase (MAPK) signaling modules. These proteins were also isolated as KLC-binding proteins and suggested to function as adaptor proteins linking cargoes to kinesin-1. ${ }^{12,13}$ JIP1 and JIP2 are highly related to each other, but are structurally unrelated to JSAP1 and JLP. In the Drosophila JIP1 mutant Aplip1, anterograde and retrograde axonal transport of vesicles is reduced. ${ }^{14}$ Moreover, loss-offunction mutations of the genes encoding Drosophila Sunday Driver (JSAP1/JIP3) or Caenorhabditis elegans UNC-16 (JSAP1/JIP3) result in mislocalized axonal cargoes, a phenotype similar to that of kinesin-1 mutants in these organisms. ${ }^{15,16}$ Although mammalian JIPs also seem to be involved in the cargo transport in axons, their roles in this transport remain fragmentary, with several studies reporting inconsistent results. No mislocalization of axonal cargoes, including mitochondria and the synaptic-vesicle marker synapsin, was detected in Jip1 and Jip2 double-knockout (dKO) neurons. ${ }^{17}$ Ablating JSAP1 in mice causes various

${ }^{1}$ Division of Molecular Cell Signaling, Cancer Research Institute, Kanazawa University, Kanazawa, Japan; ${ }^{2}$ Brain Science Institute, Saitama University, Saitama, Japan and ${ }^{3}$ Department of Biosciences, School of Science, Kitasato University, Sagamihara, Japan

${ }^{*}$ Corresponding author: K Yoshioka, Division of Molecular Cell Signaling, Cancer Research Institute, Kanazawa University, Kanazawa, Ishikawa 920-1192, Japan. Tel: +81 76234 4532; Fax: +81 76234 4532; E-mail: katsuji@staff.kanazawa-u.ac.jp

Abbreviations: APP, amyloid precursor protein; [ATP]i, intra-axonal ATP concentration; $\left[\mathrm{Ca}^{2+}\right] \mathrm{i}$, intra-axonal $\mathrm{Ca}^{2+}$ concentration; Capn2, calpain 2; cdKO, conditional double-knockout; cKO, conditional-knockout; Cyt c, cytochrome c; DAPI, 4,6-diamidino-2-phenylindole; DIV, days in vitro; dKO, double-knockout; DN, dominant-negative; d.p.i., days post-infection; DSH, dynein stalk head; Dync1h1, cytoplasmic dynein 1 heavy chain 1; eGFP, enhanced green fluorescent protein; floxed, loxP-flanked; FRET, fluorescence resonance energy transfer; frt, flippase recognition target; GFAP, glial fibrillary acidic protein; IB, isolation buffer; JIP, JNK-interacting protein; JLP, JNK-associated leucine zipper protein; JNK, c-Jun $\mathrm{NH}_{2}$-terminal kinase; JSAP1, JNK/stress-activated protein kinase-associated protein 1; KBD, KHC-binding domain; KHC, kinesin heavy chain; KLC, kinesin light chain; KO, knockout; MAPK, mitogen-activated protein kinase; Mito, mitochondrial targeting sequence; MT, microtubule; NCX, $\mathrm{Na}^{+} / \mathrm{Ca}^{2+}$-exchanger; neo, neomycin-resistance; P, postnatal day; PCR, polymerase chain reaction; PFA, paraformaldehyde; Phospho, phosphorylated; qRT-PCR, quantitative reverse-transcription PCR; SYP, synaptophysin; SYT1, synaptotagmin 1; TUNEL, terminal deoxynucleotidyl transferase-mediated dUTP nick-end labeling; VAMP2, vesicle-associated membrane protein 2

Received 21.3.14; revised 04.11.14; accepted 10.11.14; Edited by L Greene; published online 09.1.15 
developmental defects in the architecture of the brain, including a lack of telencephalic commissures, whereas no obvious abnormal accumulation of vesicles is observed in the axons. ${ }^{18-20} \mathrm{JLP}$-null mice are viable and grow normally. ${ }^{21}$ On the other hand, JIP1 and JSAP1 were recently shown to be important for the anterograde axonal transport of plasmalemmal precursor vesicles and TrkB, respectively, in rat neurons. ${ }^{22,23}$ Collectively, these data indicate that the regulation of axonal transport in mammals is complex, with JIPS mediating the transport of selective cargo. It is also possible that mammalian JIPs function redundantly, at least in part, in regulating axonal transport.

In this study, we investigated the neuronal functions of JSAP1 and JLP using in vivo and in vitro gene deletion strategies in mice. Our data establish JSAP1 and JLP as positive regulators of kinesin-1-dependent axonal transport, with functional redundancy to prevent neuronal degeneration.

\section{Results}

Conditional Jsap1 and Jlp double deletion in the dorsal telencephalon reduces growth and causes progressive neuron loss. To examine the role and importance of JSAP1 and JLP in brain development, we generated conditional deletions of Jsap1, Jlp, or both in the dorsal telencephalon using loxP-flanked (floxed) alleles of Jsap1, ${ }^{20} \mathrm{Jlp}$ (Supplementary Figure S1a), or both, in combination with the Emx1-Cre transgene. ${ }^{24}$ Region-specific Jsap1 and Jlp deletion was confirmed by immunohistochemical analysis of control mice (Jsap1 $\left.1^{f / f}: \mathrm{Jl} \mathrm{p}^{f / f}\right)$ and conditional dKO (cdKO) mice (Jsap 1/f: $: \mathrm{Jlp}^{\mathrm{f} / \mathrm{f}}:$ Emx1-Cre) at postnatal day (P) 0 (Figure 1a). Single conditional-knockout (CKO) mice (Jsap1 $1^{f / t}: J / p^{f /+}: E m x 1-$ Cre and $J \operatorname{sap}^{f /+}: J / p^{f / f}:$ Emx1-Cre) were viable and grew normally, whereas cdKO mice exhibited severe phenotypes during postnatal development. These phenotypes were distinct from those of JSAP1-null and neural cell-specific Jsap1 $1^{-/-}\left(J_{s a p} 1^{f / f}:\right.$ Nestin-Cre) mice, which die shortly after birth, most likely due to respiratory failure. ${ }^{18,20}$ The essential defect leading to this neonatal death is probably in the brainstem rather than the telencephalon, because the brainstem is responsible for the neural network that controls breathing in mammals. Although Jsap1:Jlp cdKO mice were visually indistinguishable from single Jsap1 or Jlp cKO mice at $\mathrm{P0}$, growth retardation became evident during the second postnatal week. The average weight of the cdKO mice at P14 and P21 was approximately $70 \%$ and $50 \%$, respectively, of that of single cKO or control mice (Figure 1b; Supplementary Figure S1c). Furthermore, all of the Jsap1:Jlp cdKO mice died within 5 weeks of birth (Figure 1c). These results indicate that JSAP1 and JLP are functionally redundant and have essential roles in postnatal brain development.

We further characterized Jsap1:Jlp cdKO mice through histological analyses. Coronal sections from control and cdKO mice were stained with Nissl. Control and cdKO mice were similar at PO. However, cdKO mice subsequently developed progressive ventricular enlargement (Figure 1d) that was not observed in Jsap1 cKO, Jlp cKO, or control mice at P21 (Figure 1d; Supplementary Figure S1d). In addition, Jsap1 cKO and Jsap1:Jlp cdKO mice, but not control or Jlp cKO mice, lacked the telencephalic commissure (Supplementary Figure S1e). The cerebral cortex in cdKO mice at P21 was thinner than in control mice, and there was evident neuron loss, especially in the pyramidal neurons in layer V (Figure 1e). To confirm the neuronal loss, we examined mRNA levels of Rgs8 (a layer-II/III marker) and ER81 (a layer V marker) in control and cdKO mice at P7, P14 and P21 (Figure 1f). These markers were significantly reduced in the cdKO mice compared with control mice at each age point (except for Rgs8 mRNA at P7).

We next assessed the neuronal death using immunohistochemistry. Considerable numbers of cells in the cerebral cortex and hippocampus of P21 cdKO mice were positive for active caspase-3 and terminal deoxynucleotidyl transferasemediated dUTP nick-end labeling (TUNEL), whereas very few of these apoptotic cells were found in control mice of the same age (Figure 1h; Supplementary Figures S1f). Furthermore, glial fibrillary acidic protein (GFAP) immunohistochemistry revealed the presence of reactive astrogliosis, a hallmark of central nervous system injury, in P21 cdKO mice (Supplementary Figure S1g). Taken together, these results strongly suggest that ablating the scaffold proteins JSAP1 and JLP leads to neurodegeneration.

JSAP1 and JLP are required in neurons to prevent axonal degeneration and subsequent neuronal death. To investigate the role of JSAP1 and JLP in neurons, we used a primary neuronal cell culture system in which a target gene can be disrupted using an adenovirus vector expressing Cre recombinase (AxCANCre). ${ }^{25}$ The targeted disruption of Jsap 1 and Jlp was confirmed by immunofluorescence microscopy and immunoblotting (Figure 2a). Although hippocampal neurons with a Cre-induced single Jsap1 or Jlp knockout (KO) had a normal morphology for at least 14 days after infection, the axons (stained with an anti-Tau antibody) in hippocampal neurons with Cre-induced Jsap1:Jlp dKO appeared swollen at 7 days post-infection (d.p.i.) (Figure $2 b$ ) and began to fragment at 10 d.p.i. (Figures $2 \mathrm{c}$ and d). The specificity of the Tau immunosignal was confirmed by double-immunostaining for MAP2 (a dendritic marker) and Tau (Supplementary Figure S2). Furthermore, cultures of dKO hippocampal neurons had significantly fewer cells than those of control or single Jsap 1 or Jlp KO neurons at 14 d.p.i. (Figures $2 \mathrm{e}$ and f). Although the cultures had similar numbers of cells at 10 d.p.i. (Figure 2f), the cultured dKO hippocampal neurons contained a significantly higher percentage of cells that were positive for active caspase-3 (Figures $2 \mathrm{~g}$ and $\mathrm{h}$ ). In addition, there were no significant differences in the neuronal defects between the AxCANCre-uninfected Jsap1 $1^{f / f}: J / p^{f / f}$ neurons and the Creinduced single Jsap1 or Jlp KO neurons (Figures 2d, f and $\mathrm{h}$ ), indicating that the toxicity of the adenovirus for neurons and the effect of Cre expression itself were negligible under our experimental conditions. Collectively, these results suggested that both JSAP1 and JLP are involved in regulating axonal transport, and that both genes must be deleted to impair axonal transport and cause neuron degeneration.

JSAP1 and JLP regulate kinesin-1-dependent axonal transport. We analyzed the axonal distribution of mitochondria and amyloid precursor protein (APP), which are kinesin-1 cargoes, and of synaptophysin (SYP) and synaptotagmin 1 
(SYT1), which are kinesin-3 cargoes, ${ }^{4}$ in uninfected control $\left(J\right.$ sap $1^{f / f}: \mathrm{Jlp}^{\mathrm{f} / \mathrm{f}}$ ) and AxCANCre-infected Jsap1:Jlp dKO hippocampal neurons at 7 d.p.i. All of the cargoes were almost uniformly distributed in the axons of control neurons, whereas the kinesin-1 cargoes appeared to accumulate in the swollen axons of the dKO neurons (Figures $3 a$ and $b$ ).
Indeed, kinesin-1 cargoes were present in almost all of the swollen axons, and fluorescence images showed a significantly wider distribution of kinesin- 1 than kinesin- 3 cargoes within the axons (Figure $3 \mathrm{c}$ ). These results suggested that JSAP1 and JLP selectively regulate kinesin-1-dependent axonal transport. a

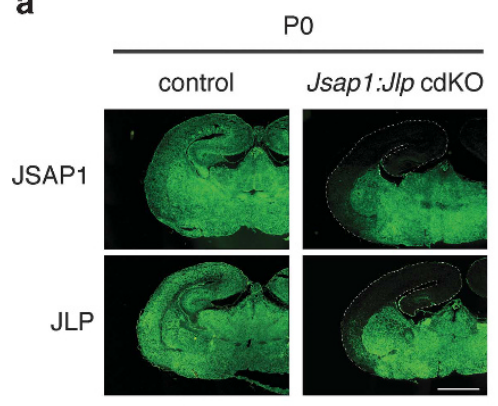

c

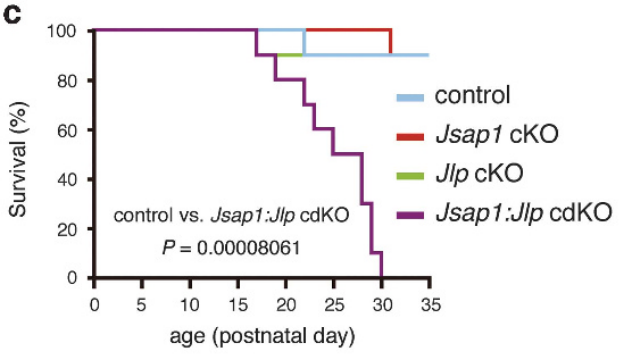

e

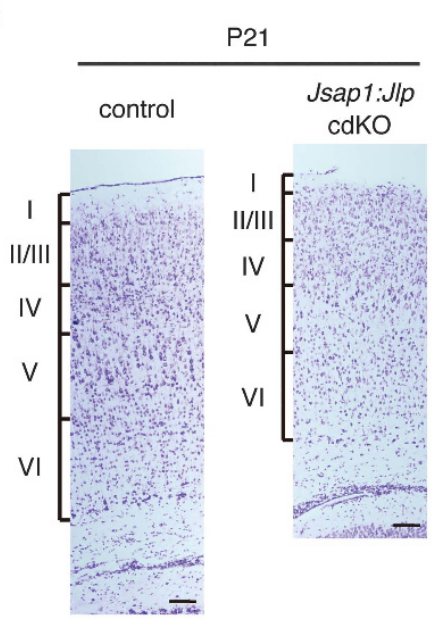

g

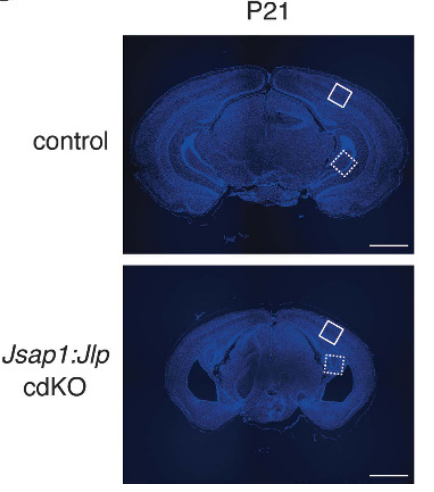

b

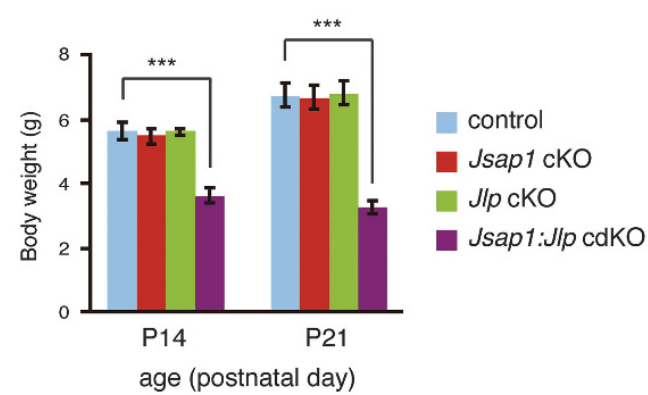

d
PO

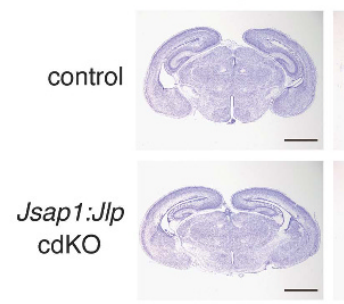

f

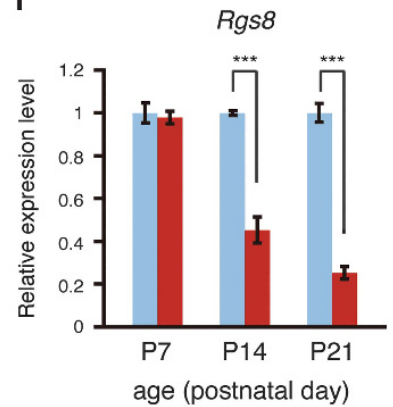

P7

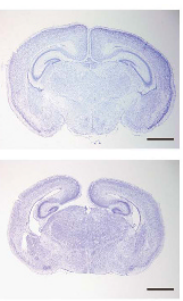

P14

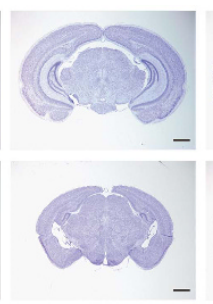

ER81 h

active

caspase-3

active

caspase-3 (black-and-white)

DAPI

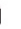

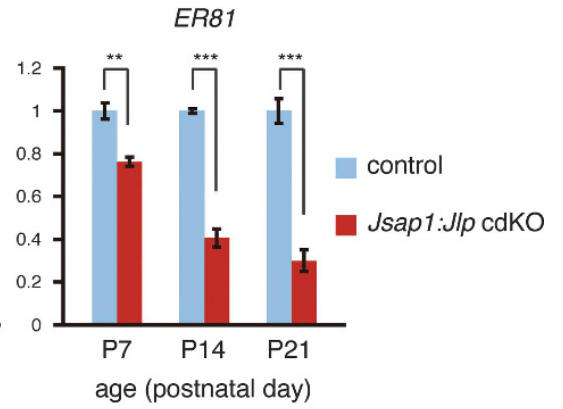

age (postnatal day)
P21

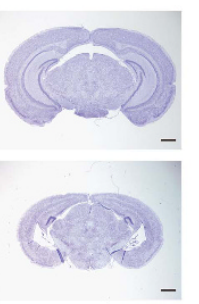

Cerebral cortex (P21)
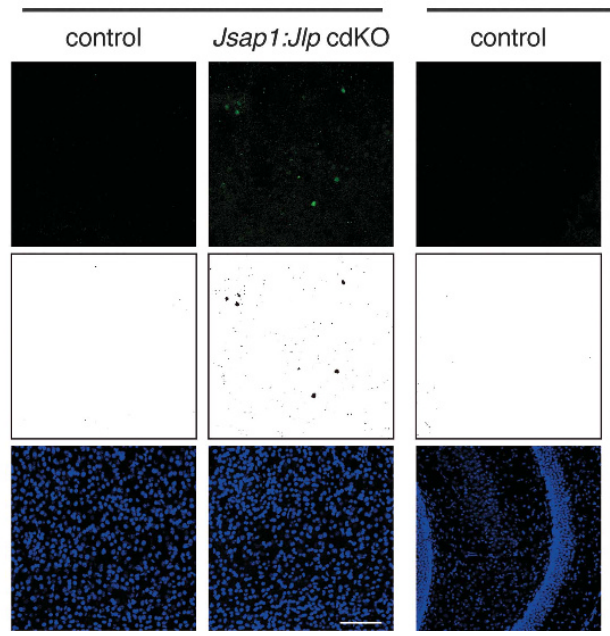

Jsap 1:Jlp cdKO
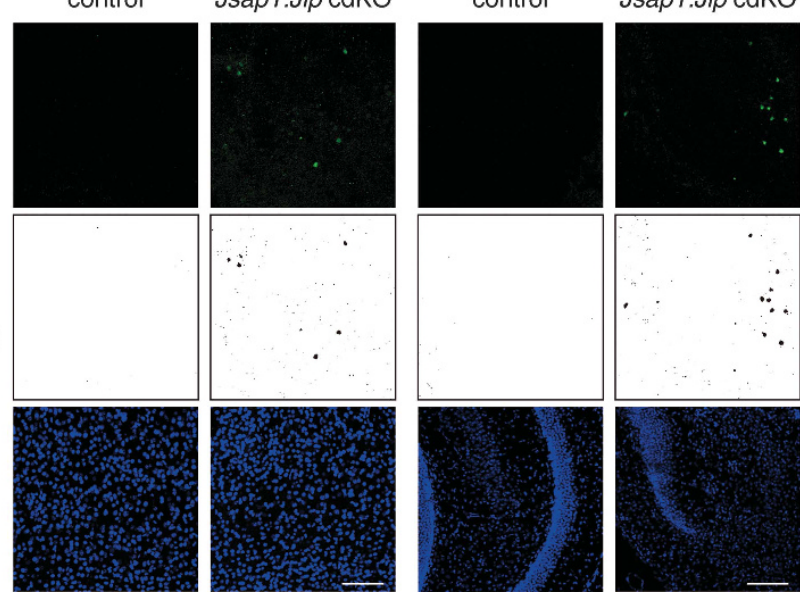
We next analyzed the movement of mitochondria and APP in the axons of primary hippocampal neurons. We used lentiviral vectors expressing enhanced green fluorescent protein (eGFP) fused to a mitochondrial targeting sequence (Mito-eGFP) or to APP (APP-eGFP), and confirmed that MitoeGFP and APP-eGFP colocalized with MitoTracker-stained mitochondria and antibody-stained APP, respectively, in the axons (Supplementary Figures $\mathrm{S} 3 \mathrm{a}$ and $\mathrm{b}$ ). We used time-lapse imaging to measure the mitochondrial and APP movement in the axons of hippocampal neurons infected with Mito-eGFP or APP-eGFP lentiviruses, in the presence or absence of AxCANCre. In AxCANCre-infected Jsap1:Jlp dKO hippocampal neurons at 3 d.p.i., anterograde-moving mitochondria and APP were significantly decreased compared with those in control hippocampal neurons; retrograde mitochondrial and APP transport were unchanged, and stationary mitochondria and APP were significantly increased in the AxCANCreinfected Jsap1:Jlp dKO hippocampal neurons compared with control neurons (Figures 4a-d; Supplementary Movies S1). Deleting JSAP1 or JLP alone did not significantly impair anterograde or retrograde mitochondrial or APP transport in hippocampal neurons (Figures $4 a-d$ and Supplementary Movies S2). We also characterized the movements of the kinesin-3 cargoes SYP and SYT1 using lentiviruses expressing SYP-eGFP and SYT1-eGFP, which mostly colocalized with endogenous vesicle-associated membrane protein 2 (VAMP2) (Supplementary Figures S3c and d). There were no significant changes in the anterograde or retrograde SYP-eGFP or SYT1eGFP transport in control versus AxCANCre-infected dKO hippocampal neurons at 3 d.p.i. (Figures $4 \mathrm{e}$ and $\mathrm{f}$; Supplementary Movies S9-S16). These results indicated that the double deletion of JSAP1 and JLP selectively impairs kinesin-1-dependent cargo transport in axons.

We conducted in vitro rescue experiments with both wildtype and altered forms of JSAP1, including a mutant that has a substitution in the leucine zipper domain and cannot interact with $\mathrm{KLC}^{13}$ and a deletion mutant lacking the KHC-binding domain (KBD). ${ }^{26}$ We used lentiviral vectors to express these wild-type and mutant JSAP1 forms as hemagglutinin (HA)-tagged proteins (designated HA-JSAP1-WT, -LZ and $-\triangle K B D)$ in primary hippocampal neurons. Although HA-JSAP1-WT, -LZ and - $\triangle K B D$ were widely distributed along the axons, double-mutant HA-JSAP1-LZ/DKBD failed to exit the cell body, as reported previously ${ }^{26}$ (Figure 5a). We next conducted a time-lapse study using Jsap1:Jlp dKO neurons infected with the various HA-JSAP1 lentiviral constructs. The expression of HA-JSAP1-WT or -LZ, but not $-\triangle \mathrm{KBD}$, prevented the impaired anterograde transport and the increased stationary mitochondria and APP observed in the axons of AxCANCre-infected neurons at 3 d.p.i. (Figure 5b; Supplementary Movies S17-S20 and S23-S25). In the dKO neurons, HA-JSAP1-WT, -LZ and $-\triangle K B D$ were widely and similarly distributed along the axons as in control neurons (Supplementary Figure S4). Furthermore, expressing HAJSAP1-WT or -LZ, but not $-\triangle K B D$, reversed the axonal degeneration and neuron loss in the dKO hippocampal neurons (Figure $5 c$ ). Similar results were obtained with HAJLP-WT or $-\triangle \mathrm{KBD}$ lentiviruses (Supplementary Figure S5; Movies S21). Collectively, these results indicated that JSAP1 and JLP regulate the anterograde axonal transport of mitochondria and APP through their interaction with the kinesin-1 KHC subunit.

JSAP1 and JLP regulate kinesin-1 binding to MTs. We next conducted MT co-sedimentation experiments to determine whether the interactions of JSAP1 and JLP with KHC affect kinesin-1's binding to MTs. We added taxol-stabilized MTs to lysates from primary cultured neurons, precipitated the MTs by centrifugation, and analyzed the MT pellets by immunoblotting. Three days after AxCANCre infection, hippocampal neurons with Cre-induced dKO contained significantly less MT-bound KHC than did AxCANCreuninfected control hippocampal neurons (Figures $6 a$ and b). Moreover, the lentiviral-mediated expression of HA-JSAP1WT and $-L Z$, but not $-\triangle K B D$, reversed the decreased MT binding seen in dKO neurons (Figures $6 a$ and b). Similar results were obtained with HA-JLP-WT and $-\triangle K B D$ lentiviruses (Supplementary Figure S6). In addition, we examined the effects of overexpressed $\mathrm{KHC}$ on $\mathrm{KHC}-\mathrm{MT}$ binding in Jsap1:Jlp dKO neurons expressing exogenous wild-type or mutant JSAP1s. The relative dKO-associated decrease in MT binding in the $\mathrm{KHC}$-overexpressing dKO neurons was smaller than that in KHC-non-overexpressing neurons (Figures $6 \mathrm{c}-\mathrm{f}$ ), probably due to a stoichiometric imbalance between $\mathrm{KHC}$ and JSAP1.

We next investigated whether kinesin-1's cargo binding was also affected in dKO neurons. As axonal mitochondria are reported to associate with $\mathrm{KHC}^{27}$ we analyzed highly enriched mitochondrial fractions from primary hippocampal neurons by immunoblotting with an anti-KHC antibody.

Figure 1 Reduced growth and premature death in Jsap1:Jlp cdKO mice. (a) Conditional Jsap1 or Jlp deletion in the dorsal telencephalon. Coronal sections from P0 Jsap ${ }^{t / f}$ : $J / p^{f f f}$ (control) and Jsap1:Jlp cdKO mice were stained with anti-JSAP1 and anti-JLP antibodies. (b) Growth retardation in Jsap1:Jlp cdKO mice. The indicated male mice were weighed at P14 and P21, ( $n=5$ mice per group). (c) Kaplan-Meier survival curves of the indicated mice ( $n=10$ mice per group). All of the Jsap1:Jlp cdKO mice died within 30 days of birth. (d) Nissl staining of coronal brain sections of control and Jsap1:Jlp cdKO mice at P0, P7, P14 and P21. The Jsap1:Jlp cdKO mice showed progressive ventricular enlargement. (e) Neuronal loss and thinning of the cerebral cortex in Jsap1:Jlp cdKO mice. Coronal sections of the visual cortex of control and cdKO mice at P21 were stained with Nissl. Neuronal loss was evident in layer V of the cdKO mice. (f) Relative mRNA levels of Rgs8 and ER81 measured by qRT-PCR in control and cdKO mice at P7, P14 and P21. The relative expression levels were normalized to Gapdh. The relative Rgs8 and ER81 mRNA levels in cdKO mice decreased in an age-dependent manner. Images were captured by a Keyence Biozero BZ-8100 microscope (Keyence, Osaka, Japan) with a 10 x objective (a and e) or an Olympus SZX10 stereomicroscope (Olympus, Tokyo, Japan) (d), and were used to create composite images. (g) Control and Jsap1:Jlp cdKO mouse brains at P21 stained with DAPI. For each picture, four wide-field images captured using a fluorescence microscope with a $2 \times$ objective lens (BZ-9000; Keyence) were combined to generate an overall image of the brain, using the image-joiner function in the BZAnalyzer software (Keyence). Areas in the solid- and dotted-lined boxes represent the cerebral cortex and hippocampus, respectively, which were immunohistochemically analyzed for active caspase-3 in h. (h) Coronal brain sections from control and Jsap 1:Jlp cdKO mice were stained with an anti-active caspase-3 antibody and DAPI. Considerable numbers of active caspase-3-positive cells were detected in the cerebral cortex and hippocampus of Jsap1:Jlp cdKO mice, but not in those of control mice. The images were captured by a Zeiss LSM510 META confocal microscope with a $20 \times$ objective. In addition to the color images for active caspase-3, the corresponding black-and-white binary images are included for better visualization. ${ }^{* \star} P<0.01 ;{ }^{* \star \star} P<0.001$. Scale bars, $1 \mathrm{~mm}(\mathbf{a}, \mathbf{d}$ and $\mathbf{g}) ; 100 \mu \mathrm{m}(\mathbf{e}$ and $\mathbf{~})$ 

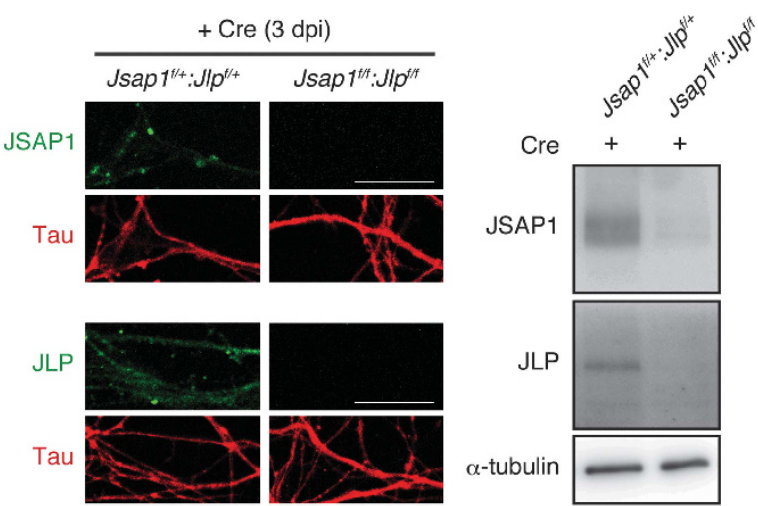

c
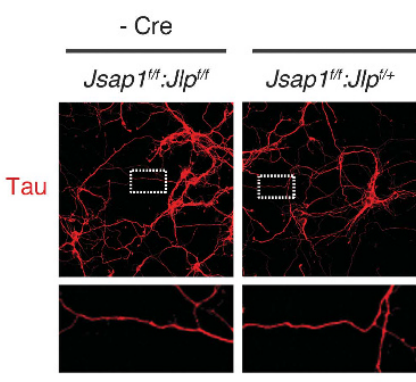

Jsap $1 \mathrm{KO}$

+ Cre (10 dpi)

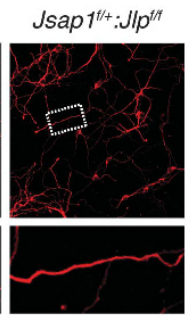

$J / p \mathrm{KO}$

e
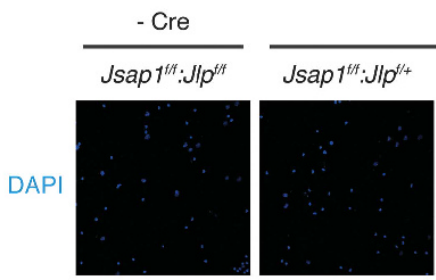

Jsap1 KO
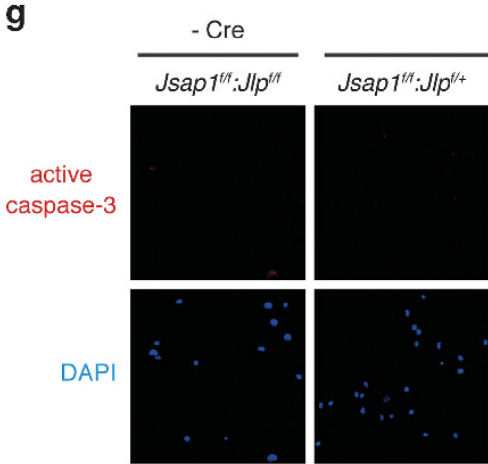

g

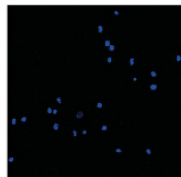

Jsap1 KO
+ Cre (14 dpi)

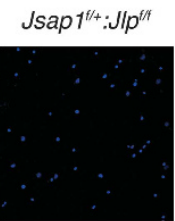

Jip KO

Jsap $^{t / 4}: \mathrm{Jlp}^{t / 1}$

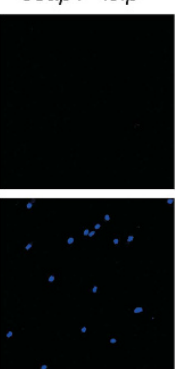

Jlp KO
Jsap 1:Jlp dKO

Jsap $1^{t / t}: \mathrm{Jl} p^{\text {tht }}$

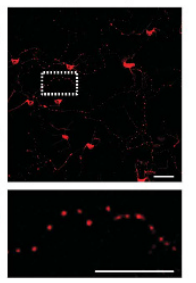

b
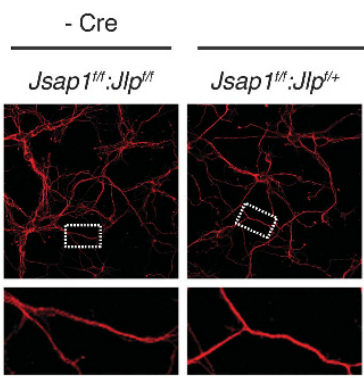

Jsap 1 KO
+ Cre (7 dpi)

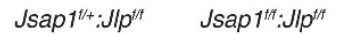
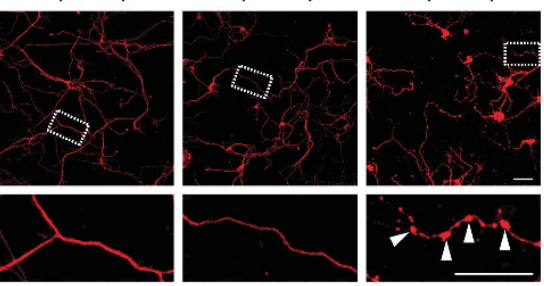

Jsap1:Jlp dKO d

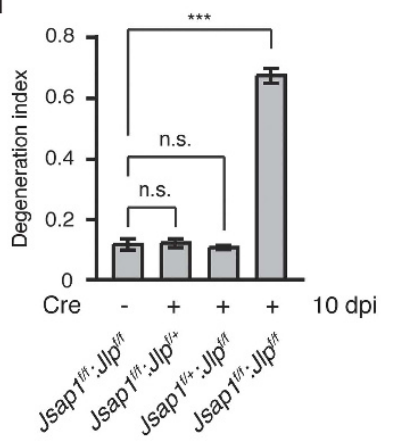

$\mathbf{f}$
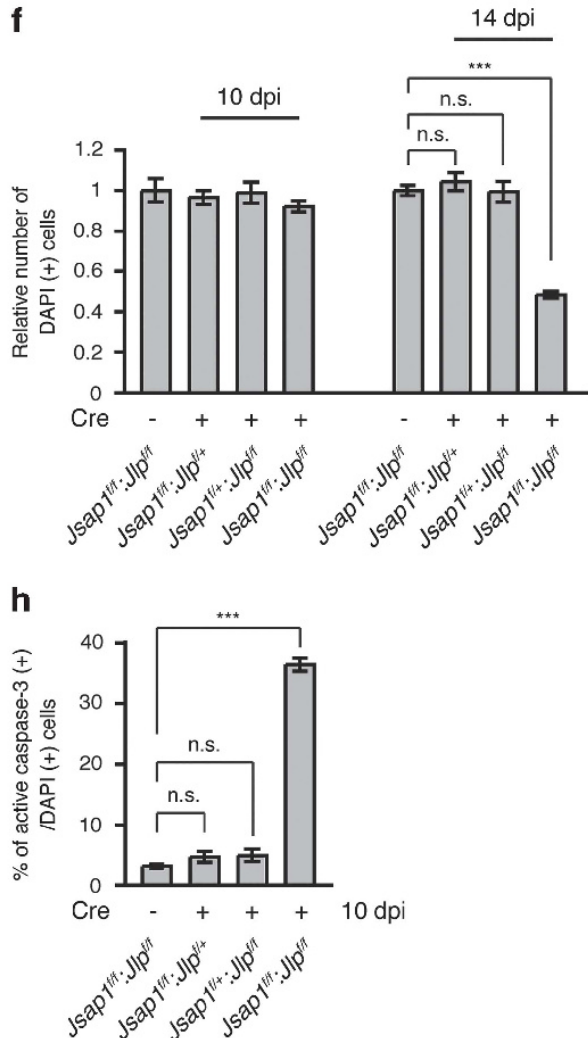

Figure 2 Loss of JSAP1 and JLP causes axonal degeneration and subsequent neuronal death. (a) Cre-induced deletion of Jsap1 and Jlp in primary hippocampal neurons. Immunofluorescence microscopy (left) and immunoblotting (right) of $\mathrm{Jsap}^{t /+}: \mathrm{Jl} \mathrm{p}^{t /+}$ and $\mathrm{Jsap} 1^{t / f}: \mathrm{Jl} \mathrm{p}^{t / f}$ hippocampal neurons infected with AxCANCre were performed using antiJSAP1 and anti-JLP antibodies at 3 d.p.i. Tau staining was included as an axonal marker (left), and $\alpha$-tubulin was used as a loading control (right). (b-h) Axonal swelling (b), axonal degeneration (c and d) and neuronal death (e-h) in Jsap1:Jlp dKO hippocampal neurons. Uninfected Jsap $1^{f / f}$ :Jlp/t, single Jsap1 or Jlp KO, and Jsap1:Jlp dKO hippocampal neurons at the indicated d.p.i. with AxCANCre were stained with anti-Tau antibody (b and c), DAPI (e and $\mathbf{g}$ ) and anti-active caspase-3 antibody (g). Arrowheads in $\mathbf{b}$ indicate swollen axons. Lower panels in $\mathbf{b}$ and $\mathbf{c}$ show higher magnifications of the boxed areas in the upper panels. The results in $\mathbf{c}$ were quantified (d). The extent of axonal degeneration was determined by calculating the degeneration index as described in Materials and methods section. Numbers of DAPI- (f) and active caspase-3-positive (h) cells were quantified. ${ }^{* * *} P<0.001$; NS, not significant. Scale bars, $50 \mu \mathrm{m}$ 
At 3 d.p.i., there was no reduction of $\mathrm{KHC}$ in the AxCANCreinfected dKO neurons relative to that in AxCANCre-uninfected (control) hippocampal neurons (Figure 6g). Together, these results indicated that JSAP1 and JLP positively regulate kinesin-1's binding to MTs through their interaction with $\mathrm{KHC}$, most likely without affecting interactions between kinesin-1 and its cargoes.

Loss of JSAP1 and JLP raises the intra-axonal $\mathrm{Ca}^{2+}$ concentration. Mitochondria move within the axon and to areas with high energy demands, and provide ATP. Several reports have suggested that a failure of $\mathrm{Na}^{+} / \mathrm{K}^{+}$-ATPase because of energy deficits increases the net influx of $\mathrm{Na}^{+}$; this reverses the $\mathrm{Na}^{+} / \mathrm{Ca}^{2+}$-exchanger (NCX), causing $\mathrm{Ca}^{2+}$ to accumulate in axons, and leading eventually to axon degeneration. ${ }^{28}$ In addition, it is possible that arrested mitochondria in axons are unable to maintain their normal function, resulting in a loss of ATP generation and energy deficiency. We first asked whether the ATP level was altered in the axons of Jsap 1:Jlp dKO primary neurons, using a lentiviral vector for ATeam, a fluorescence resonance energy transfer (FRET)-based ATP indicator. ${ }^{29}$ We detected a significantly lower [ATP]i (intra-axonal ATP concentration) at 5 d.p.i. in the axons of AxCANCre-infected Jsap1:Jlp dKO neurons than in those of AxCANCre-uninfected (control) $\mathrm{Jsap}^{\mathrm{f} / \mathrm{f}}: \mathrm{Jl} \mathrm{p}^{\mathrm{f} / \mathrm{f}}$ neurons (Supplementary Figure S7). We next analyzed changes in $\left[\mathrm{Ca}^{2+}\right] \mathrm{i}$ (intra-axonal $\mathrm{Ca}^{2+}$ concentration) using a lentiviral vector for G-CaMP7, a genetically encoded $\mathrm{Ca}^{2+}$ indicator. ${ }^{30}$ We observed a substantial increase in $\left[\mathrm{Ca}^{2+}\right] \mathrm{i}$ at 5 d.p.i. in the AxCANCre-infected Jsap1:Jlp dKO neurons, but not in single Jsap1 or Jlp KO or AxCANCreuninfected (control) Jsap1 $1^{f / f}: J / p^{f / f}$ neurons (Figures 7a and b). The increased $\left[\mathrm{Ca}^{2+}\right]$ i was almost completely abolished when dKO hippocampal neurons were cultured in the presence of bepridil, an NCX inhibitor (Figures $7 a$ and b). Interestingly, we also observed substantial JNK activation in the axons of AxCANCre-infected dKO neurons at 5 d.p.i. (Figures 7c and d). Treating these cells with bepridil effectively blocked JNK activation (Figures 7c and d), although treatment with SP600125, a JNK inhibitor, did not suppress the elevation in $\left[\mathrm{Ca}^{2+}\right] \mathrm{i}$ (Figures $7 \mathrm{a}$ and $\mathrm{b}$ ). Taken together, these results indicated that the ablation of JSAP1 and JLP causes intraaxonal $\mathrm{Ca}^{2+}$ accumulation, most likely through NCX, leading to JNK hyperactivation.

a

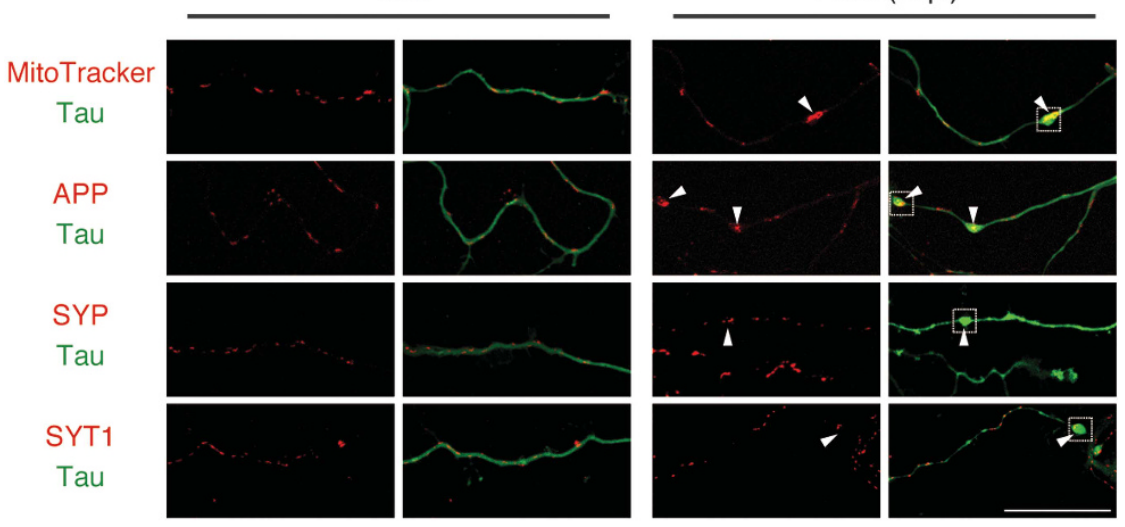

b

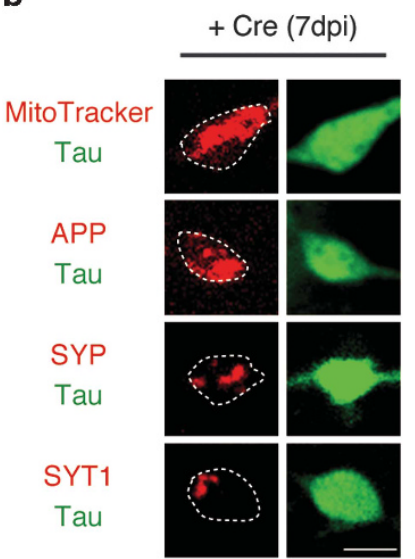

C

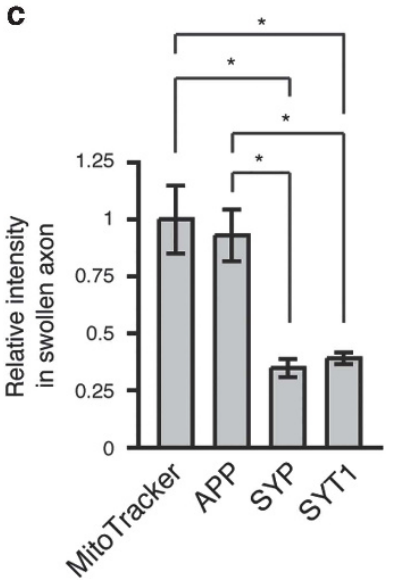

Figure 3 Kinesin-1 cargoes are selectively accumulated in the swollen axons of Jsap1:Jlp dKO neurons. (a) Fluorescence microscopy of uninfected or AxCANCre-infected $\mathrm{Jsap}^{t f f}: \mathrm{J} / p^{f f f}$ hippocampal neurons at 7 d.p.i., using MitoTracker (for mitochondria) and the indicated antibodies. Accumulations of mitochondria and APP (kinesin-1 cargoes), but not SYP or SYT1 (kinesin-3 cargoes), were detected in the swollen axons of Jsap1:Jlp dKO neurons. Axons were marked by Tau staining. Arrowheads indicate swollen axons. (b) Higher magnifications of the boxed areas in a. Swollen axons are outlined by dotted lines. (c) The ratio of the mean immunofluorescence intensity (per unit area of each swollen axon) for MitoTracker, APP, SYP or SYT1 relative to that of Tau in $\mathbf{b}$. ${ }^{*} P<0.05$. Scale bars, $50 \mu \mathrm{m}$ (a); $5 \mu \mathrm{m}$ (b) 
a

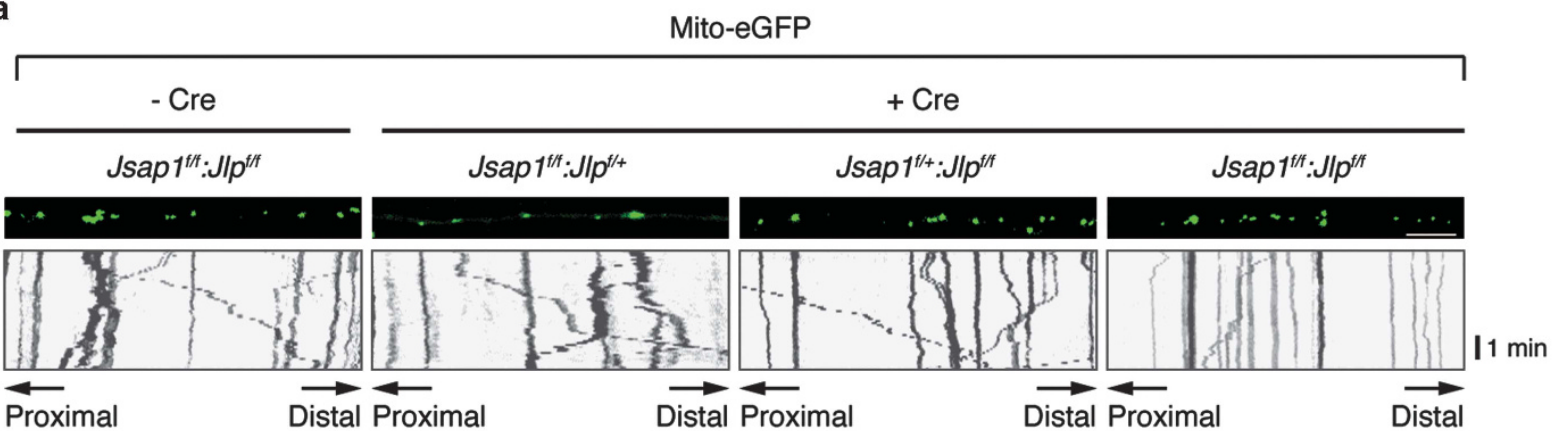

b

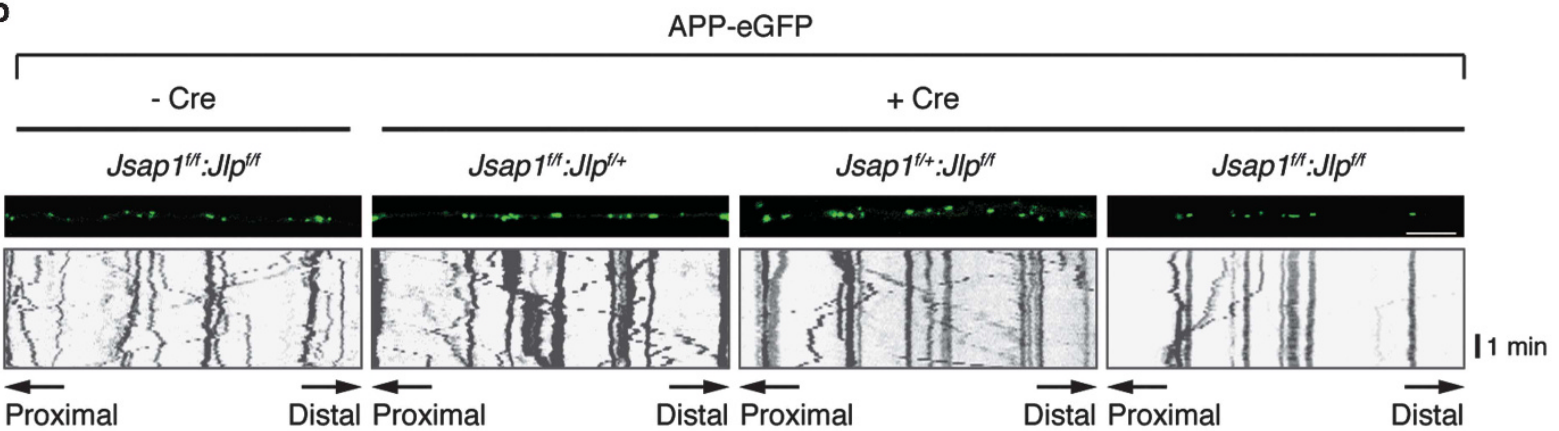

C

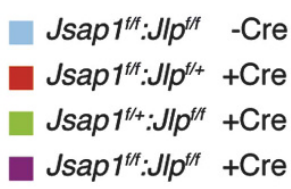

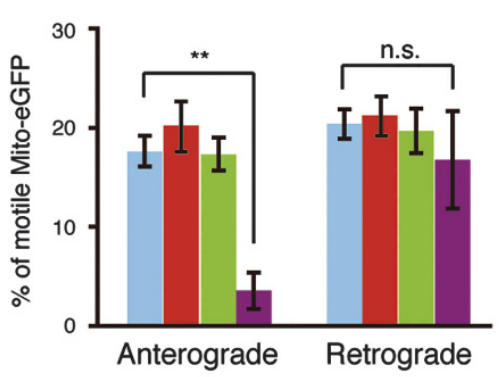

e
Usap $1^{f / f}: J / p^{f / f} \quad-$ Cre
usap $1^{t / f}: J / p^{f /+}+$ Cre
Jsap $1^{f / *}: J / p^{f / t}+$ Cre

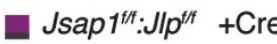
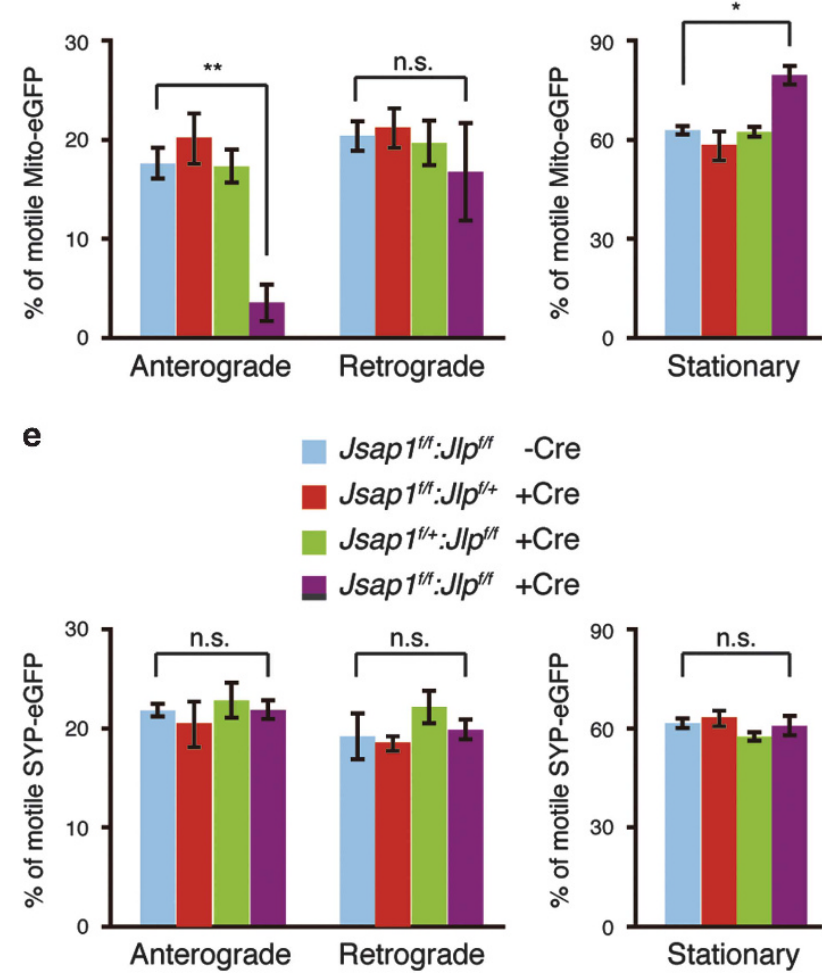

d
Jsap $1^{f f f}: J 1 p^{f / f} \quad-$ Cre
- Jsap $1^{f / f}: J l p^{f /+}+$ Cre
Jsap $1^{f / 4}: J / p^{f / t}+$ Cre

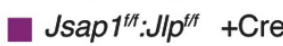
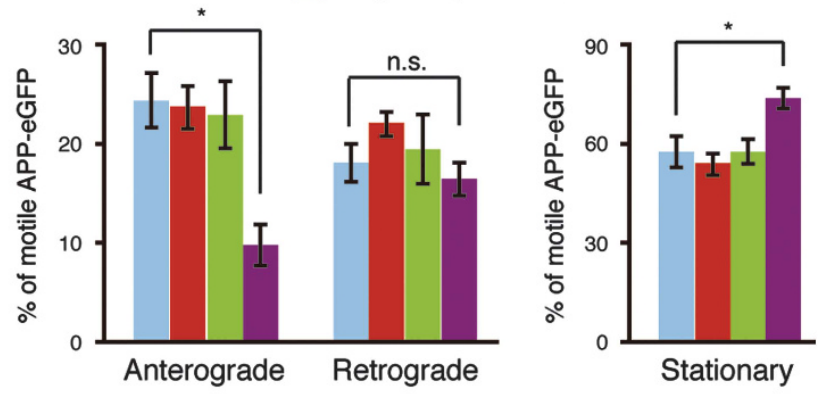

f
Jsap $1^{t / f}: J l p^{f / t} \quad-C r e$
Jsap $1^{f / t}: \mathrm{Jlp}^{\mathrm{f} /+}+\mathrm{Cre}$
Jsap $1^{f /+}:$ Jlp $^{f / t}+$ Cre

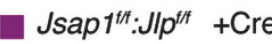
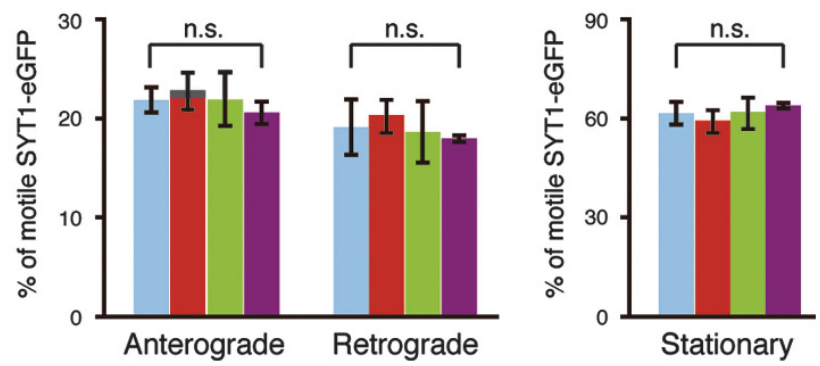
We further investigated whether the $\mathrm{Ca}^{2+}$-dependent protease calpain was activated in dKO primary neurons, as calpain is reported to be involved in axonal degeneration and neuronal death. ${ }^{31}$ Immunoblotting with an antibody against $a$-spectrin, a calpain substrate, indicated the presence of proteolytic $a$-spectrin fragments in cell lysates prepared from a

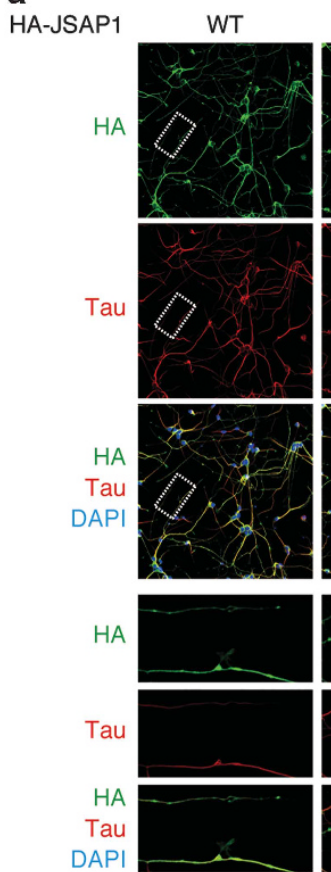

LZ
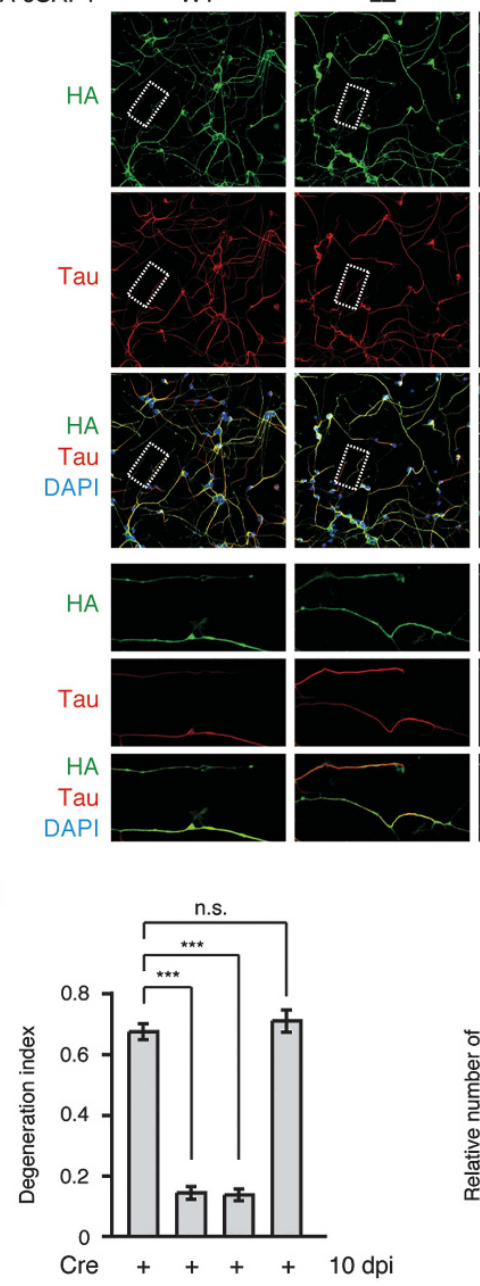

HA-JSAP1 - WT LZ $\triangle K B D$ $\triangle \mathrm{KBD}$
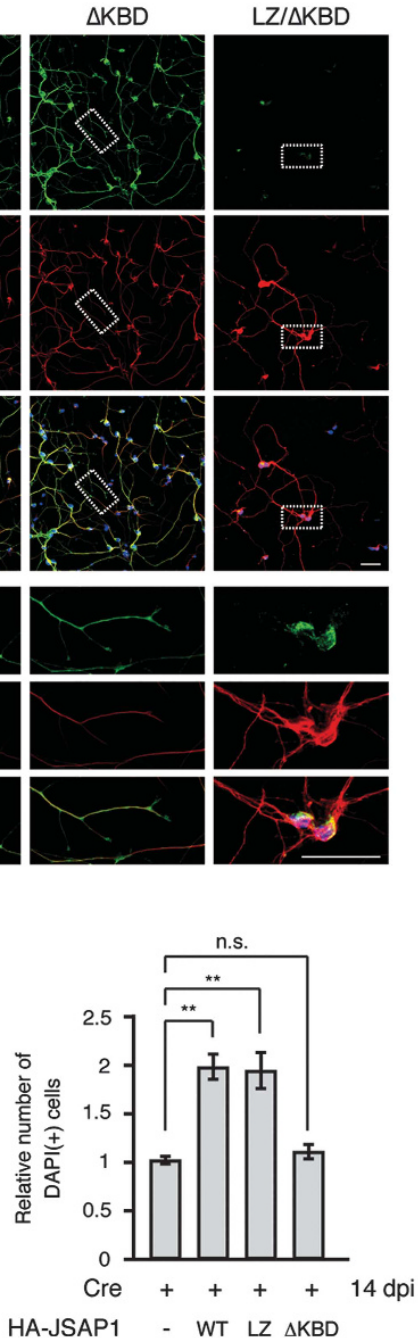

b
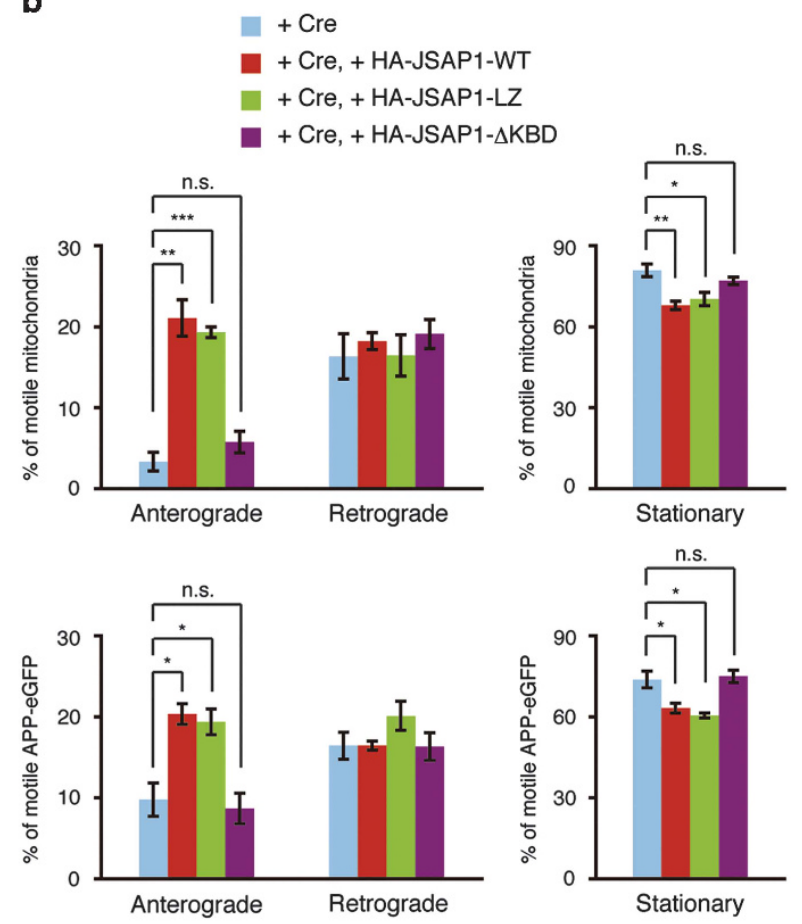

Figure 5 Axonal transport defects are reversed by JSAP1-WT and -LZ, but not - $\triangle \mathrm{KBD}$. (a) Jsap $1^{f /+}: \mathrm{Jl} p^{f /+}$ hippocampal neurons with lentiviral expression of HA-JSAP1-WT, $-L Z,-\triangle K B D$ and $-L Z / \triangle K B D$, stained with DAPI and antibodies against HA and Tau as indicated. Bottom panels: higher magnifications of the boxed areas in the top panels. HAJSAP1-WT, -LZ and - $\triangle K B D$, but not -LZ/ $\triangle K B D$, were uniformly distributed in the neurons. (b) Time-lapse study and quantitative analysis of mitochondria and APP, performed as in Figure $4 a$ and d, with AxCANCre-infected Jsap $1^{t / f}$ :Jlp $p^{f / f}$ hippocampal neurons and HA-JSAP1 or APP-eGFP lentiviruses as indicated. Mitochondria were stained with MitoTracker. See also Supplementary Movies S17-S20 and S23-S25. HA-JSAP1-WT and -LZ, but not - $\triangle$ KBD, reversed the defective anterograde movement of mitochondria and APP and the accumulation of stationary mitochondria and APP in the Jsap1:Jlp dKO neurons (Figures 4a-d). (c) Axonal degeneration (left) and neuronal loss (right) in Jsap1:Jlp dKO neurons were rescued by HA-JSAP1-WT and -LZ, but not $-\triangle \mathrm{KBD}$. Immunofluorescence microscopy and quantitative analysis were performed as in Figures $2 c-f$, using AxCANCre-infected Jsap ${ }^{1 / f}$ :JI $p^{t / f}$ hippocampal neurons with HA-JSAP1 lentiviruses as indicated. ${ }^{*} P<0.05 ;{ }^{* *} P<0.01$; ${ }^{* * *} P<0.001$; NS, not significant. Scale bars, $50 \mu \mathrm{m}$

Figure 4 JSAP1 and JLP regulate kinesin-1-dependent axonal transport. (a) Kymograph analysis of lentivirally expressed Mito-eGFP in hippocampal neurons. Time-lapse imaging of uninfected or AxCANCre-infected $J$ sap $1^{f f}: J p^{t /+}$, Jsap $1^{t /+}: J p^{t / f}$, and $J$ sap $1^{t / f}: J I p^{f / t}$ hippocampal neurons expressing Mito-eGFP at 3 d.p.i. Upper panels: opening frames from live-imaging movies (see Supplementary Movies S1-S4). Lower panels: kymographs generated from the movies. Time is on the $y$ axis, and distance is on the $x$ axis. Proximal to distal indicates the soma to axon terminal direction. (b) Analysis of APP-eGFP motility as in a. Upper panels: opening frames from live-imaging movies (see Supplementary Movies S5-S8). Lower panels: kymographs generated from the movies. (c) Quantification of the results in a. In Jsap1:Jlp dKO neurons, anterograde but not retrograde mitochondrial movement was significantly impaired, and the number of stationary mitochondria was significantly increased. (d) Quantification of the results in $\mathbf{b}$. As with mitochondria, anterograde APP movement was defective in dKO neurons, and stationary APP was increased. (e and f) SYP-eGFP (e) and SYT1-eGFP (f) motility, analyzed as in a (see also Supplementary Movies S9-S16). Motile and stationary SYP and SYT1 did not differ significantly between the neurons. ${ }^{*} P<0.05$; ${ }^{* *} P<0.01$; NS, not significant. Scale bars, $10 \mu \mathrm{m}$ 
AxCANCre-infected dKO hippocampal neurons at 5 d.p.i. (Figure 7e). This $a$-spectrin cleavage was fully sensitive to MDL28170, a potent inhibitor of calpain 1 and 2, but was not sensitive to SP600125 (Figure 7e). Consistent with observations in Jsap 1:Jlp dKO primary neurons, calpain and JNK were more active in Jsap 1:Jlp cdKO than in control mice at P14 and
P21 (Figure 7f). Treating Jsap1:Jlp dKO neurons with MDL28170 or SP600125 reversed the axonal degeneration and neuronal loss to levels comparable to those in control neurons (Figure $7 \mathrm{~g}$ ). Collectively, these results indicated that JNK mediates axonal degeneration and neuronal death in cooperation with calpain in Jsap 1:Jlp dKO neurons. a

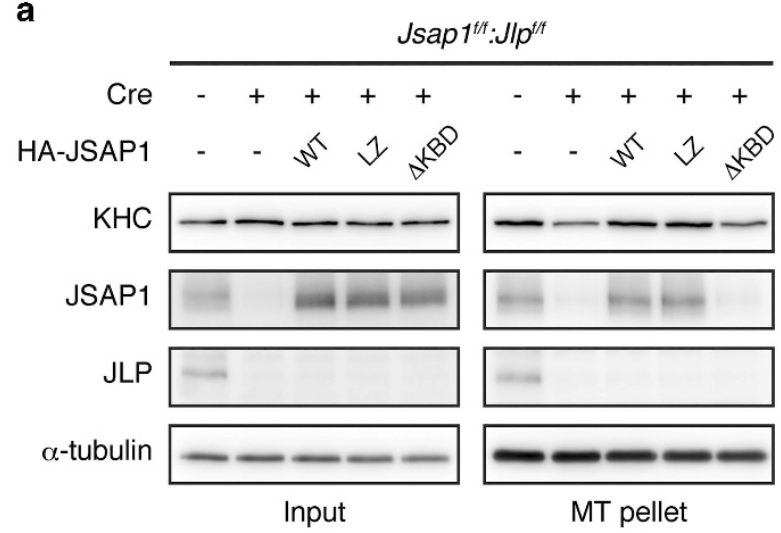

C

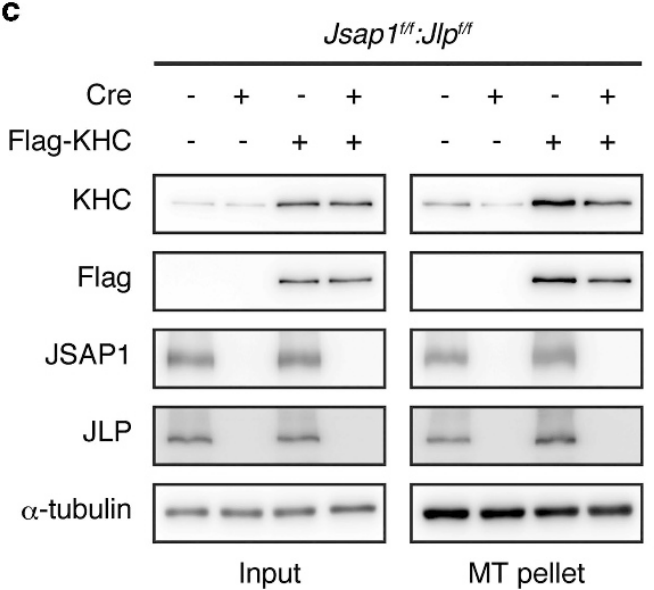

e

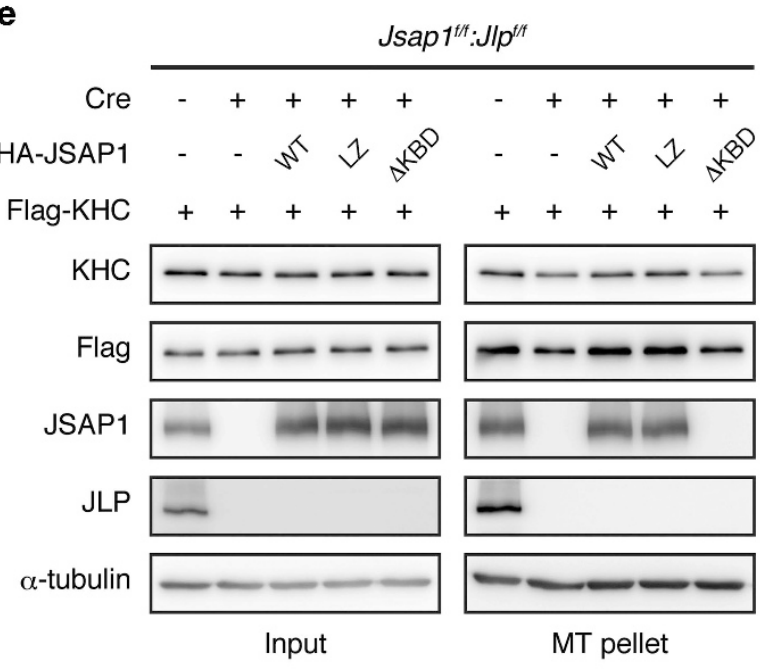

b

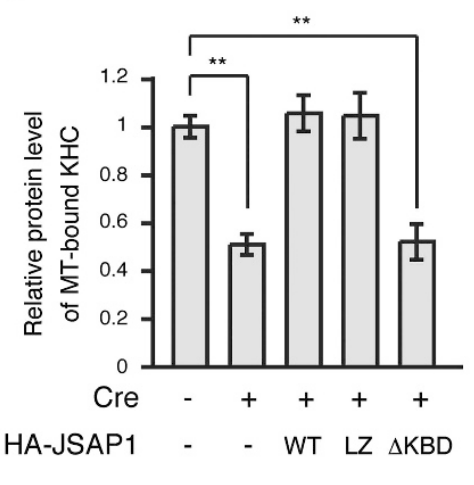

d

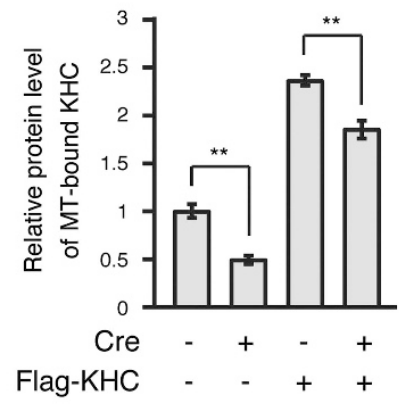

f

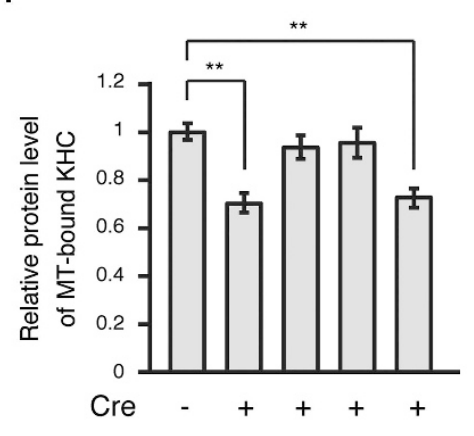

HA-JSAP1 - - WT LZ $\triangle K B D$

Flag-KHC +++++ g

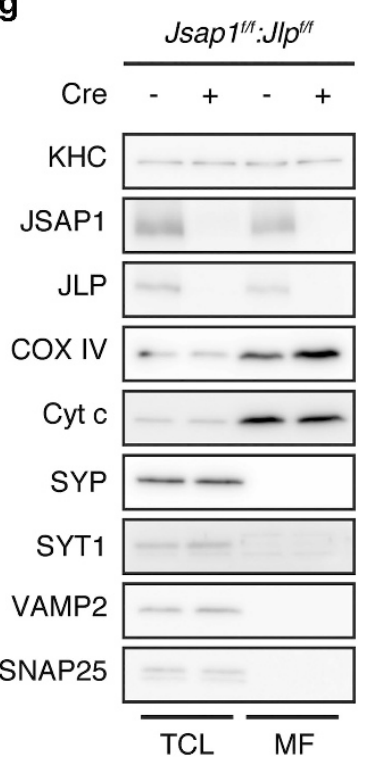


Retrograde axonal transport of activated JNK is required for neuronal death. The nuclear phosphorylated (Phospho) c-Jun levels were markedly higher in the AxCANCre-infected Jsap 1:Jlp dKO hippocampal neurons than in control or single Jsap1 or Jlp KO hippocampal neurons at 7 d.p.i., and the number of Phospho-c-Jun-positive cells increased even more at 10 d.p.i. (Figures $8 \mathrm{a}$ and b). However, activated JNK was not detected in the nucleus of these neurons at any time point. The neuronal loss seen in Jsap1:Jlp dKO neurons was almost completely blocked by the lentiviral vector-mediated expression of a dominant-negative (DN) c-Jun fused to eGFP (eGFP-DN-c-Jun) (Figure 8c). As JNK is responsible for phosphorylating and activating the transcription factor C-Jun, ${ }^{32}$ activated axonal JNK in Jsap1:Jlp dKO neurons may relocate to the nucleus and promote neuronal death by phosphorylating and activating nuclear c-Jun. To examine this possibility, we used lentiviral vectors to express eGFP, or eGFP fused to the dynein stalk head (DSH) of cytoplasmic dynein 1 heavy chain 1 (Dync1h1) (eGFP-DSH), in Jsap 1/f; $J / p^{f / f}$ hippocampal neurons, and assessed the neuronal loss induced by AxCANCre infection. As shown in Figure 8d, both eGFP-DSH and eGFP were widely and similarly distributed in the axons. The expression of eGFP-DSH, but not eGFP, almost completely suppressed the neuron loss and the increase in Phospho-c-Jun-positive cells in the dKO neurons (Figure 8e). These results suggested that activated axonal JNK moves retrogradely in a dynein-dependent manner, and induces neuronal death by activating c-Jun in the nucleus.

\section{Discussion}

This study demonstrates for the first time that JSAP1 and JLP are functionally redundant in postnatal brain development, and that their absence causes defects such as growth retardation and progressive neuron loss. These defects were observed in Jsap1:Jlp cdKO mice, but not in Jsap1 or Jlp single cKO mice (Figure 1). The functional overlap between JSAP1 and JLP was also observed in cultured primary neurons, where the axonal degeneration and subsequent neuronal death of the Jsap1:Jlp dKO neurons were completely rescued by expressing wild-type JSAP1 or JLP, but not by JSAP1 or JLP mutants lacking KBD (Figures 2 and 5; Supplementary Figure S5). Thus, JSAP1 and JLP most likely exert their overlapping functions through interaction with kinesin-1 KHC.
Previous studies have shown that the transport of mitochondria and APP in axons occurs in association with kinesin$1 \mathrm{KHC}$ and KLC, respectively. ${ }^{33,34}$ Our time-lapse study, however, revealed that both cargoes were affected by defective anterograde axonal transport when JSAP1 and JLP were ablated (Figures $4 a-d$ ), and that these defects were reversed by exogenously expressed WT JSAP1 or JLP, but not by $\triangle$ KBD-mutant JSAP1 or JLP (Figure $5 \mathrm{~b}$; Supplementary Figure S5b). In addition, expressing JSAP1-LZ, a JSAP1 mutant that is unable to interact with KLC, almost completely rescued the impairment of anterograde axonal APP and mitochondrial transport (Figure $5 b$ ). Furthermore, there was no reduction in $\mathrm{KHC}$ in highly enriched mitochondrial fractions from Jsap1:Jlp dKO neurons (Figure 6g). Thus, it is unlikely that JSAP1 and JLP regulate the axonal transport of kinesin-1 cargoes by modulating interactions between kinesin- 1 and its cargoes. On the other hand, our MT-binding experiments indicated that JSAP1 and JLP positively regulate kinesin-1 binding to MTs through their interaction with KHC (Figures 6a and b; Supplementary Figure S6). JSAP1 and JLP may also increase kinesin-1 motor activity by enhancing kinesin-1-MT binding. Indeed, using an in vitro motility assay, Sun et al. ${ }^{26}$ showed that JSAP1 binding to KHC enhances KHC's motility along MTs. We found that the kinesin-1-MT binding was reduced in AxCANCre-infected Jsap1:Jlp dKO neurons at 3 d.p.i. (Figures $6 a$ and b), at a time point when no axon abnormalities other than defective transport could be observed, and no morphological changes or disruption of $\mathrm{Ca}^{2+}$ homeostasis were detected. Thus, we conclude that the binding of JSAP1 or JLP to KHC is a critical step in the regulation of kinesin-1-dependent axonal transport, and that its impairment is likely to be causative for axonal degeneration and neuronal death.

Scaffold proteins of mammalian MAPK cascades enable the efficient activation of specific MAPK pathways by increasing the local concentration of signaling components. ${ }^{35,36}$ In this study, however, elevated JNK was found in Jsap1:Jlp dKO neurons and mice (Figure 7). As described below, ablating the scaffold proteins JSAP1 and JLP itself would not directly cause the activation of JNK. Instead, it is likely that the depletion of JSAP1 and JLP indirectly contributed to JNK activation.

Our data indicate that the depletion of JSAP1 and JLP impaired $\mathrm{Ca}^{2+}$ homeostasis and increased $\left[\mathrm{Ca}^{2+}\right]$, which in

\footnotetext{
Figure 6 Reduced kinesin-1-MT binding, and its effect on KHC's association with mitochondria. (a) MT co-sedimentation experiments and subsequent immunoblotting were performed at 3 d.p.i. with AxCANCre-infected or -uninfected Jsap $1^{t / f}$ : Jlp $p^{t / t}$ hippocampal neurons with HA-JSAP1 lentiviruses as indicated. Decreases in endogenous KHC were detected using an anti-KHC antibody in an MT pellet prepared from neurons infected with AxCANCre alone. The reduction in KHC was reversed by lentivirally expressed HAJSAP1-WT or -LZ, but not - $\triangle$ KBD. JSAP1 and JLP protein levels in MT pellets were assessed using anti-JSAP1 and anti-JLP antibodies. Note that there was very little JSAP1 in the MT pellets from Jsap1:Jlp dKO neurons that did not express HA-JSAP1 or that expressed HA-JSAP1- $\triangle K B D$. (b) Quantification of MT-bound KHC in a. (c) Effect of overexpressed KHC on KHC-MT binding in Jsap1:Jlp dKO neurons expressing exogenous wild-type or mutant JSAP1s. The MT co-sedimentation experiments and subsequent immunoblotting were performed as in a. Decreases in $\mathrm{KHC}$ were detected using anti-KHC and anti-Flag antibodies in MT pellets prepared from neurons infected with AxCANCre in the presence or absence of Flag-KHC. (d) Quantification of MT-bound KHC in c. The relative decrease in MT binding in KHC-overexpressing Jsap1:Jlp dKO neurons (approximately 20\% reduction) was smaller than that in KHC-non-overexpressing Jsap1:Jlp dKO neurons (approximately 50\% reduction). (e) The reduction in KHC was reversed by lentivirally expressed HA-JSAP1-WT or -LZ, but not $-\triangle \mathrm{KBD}$, in the KHC-overexpressing dKO neurons. Serial infections with Flag-KHC and HA-JSAP1 lentiviruses were performed at 1 and 2 DIV, respectively. (f) Quantification of the MT-bound KHC in e. ${ }^{* *} P<0.01$. (g) AxCANCre-infected or -uninfected Jsap $1^{f / f}: J / p^{f / f}$ hippocampal neurons were used as indicated. Highly enriched mitochondrial fractions (MF) were prepared from the respective neurons 3 days after AxCANCre infection. The purity of the mitochondria in the MF was confirmed by immunoblotting with antibodies against COX IV and Cyt c (mitochondrial marker proteins), and with SYP, SYT1, VAMP2 and SNAP25 (non-mitochondrial marker proteins). Similar amounts of KHC were detected in the MF prepared from AxCANCre-infected or -uninfected Jsap $1^{t / f}$.JIp ${ }^{t / f}$ neurons using an anti-KHC antibody. TCL, total cell lysates
} 
turn triggered JNK and calpain activation in neurons. The $\mathrm{Ca}^{2+}$-dependent protease calpain may have a key role in neurodegeneration. ${ }^{31} \mathrm{~A}$ number of neuronal calpain substrates have been identified, although substrates relevant to neurodegeneration are poorly understood. We used inhibitors to show that JNK and calpain are involved in regulating the axonal degeneration and subsequent neuronal death in Jsap 1:Jlp dKO neurons (Figure 7g). It should be noted a

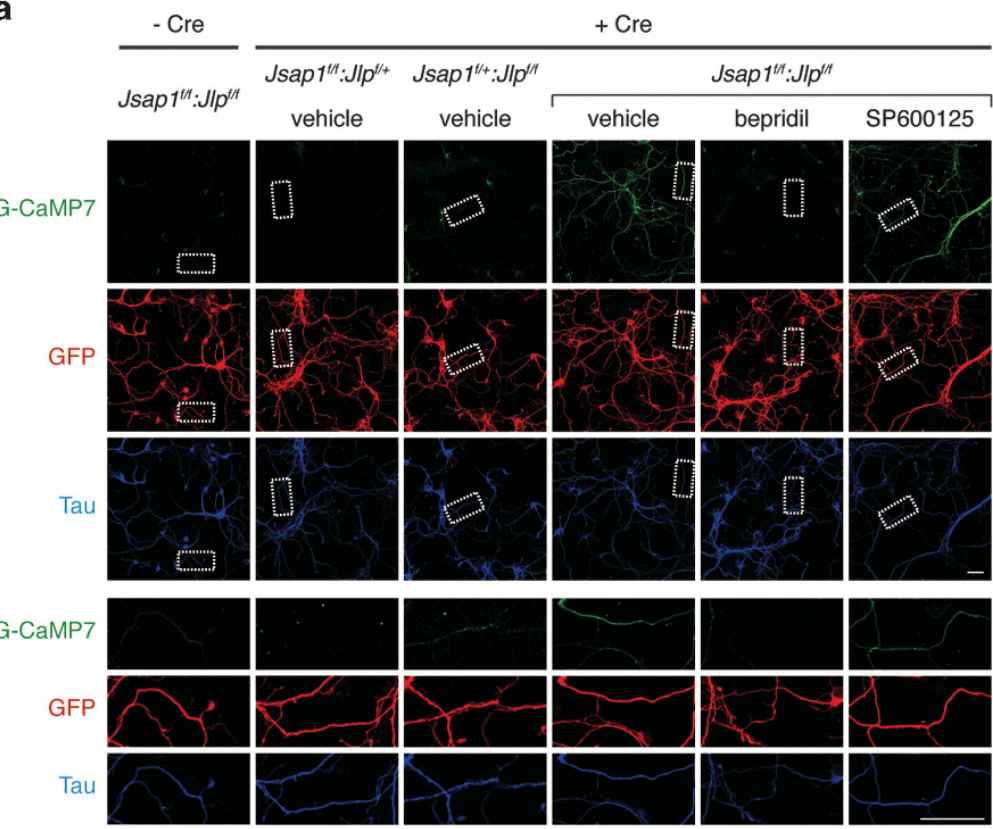

c

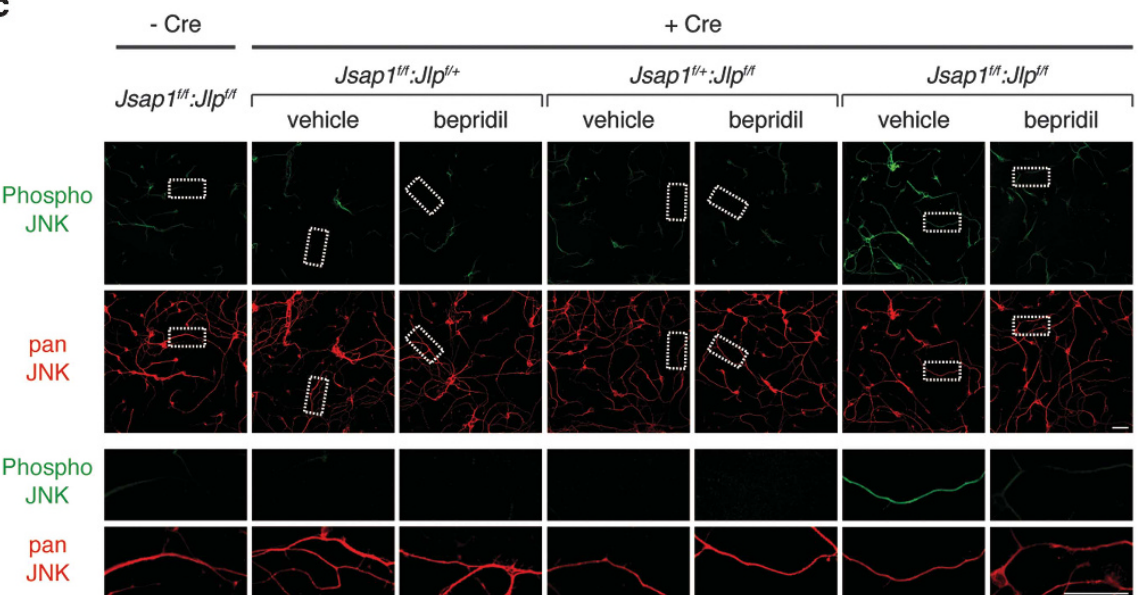

b

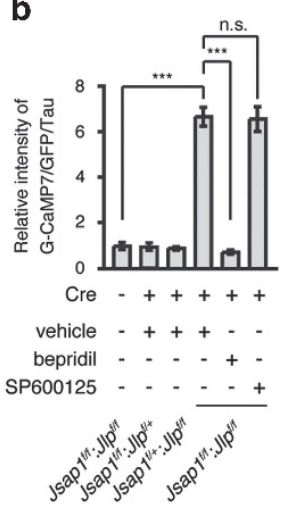

d

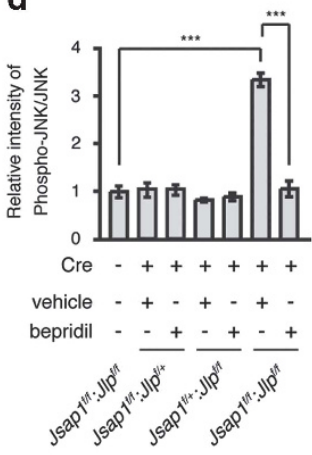

e

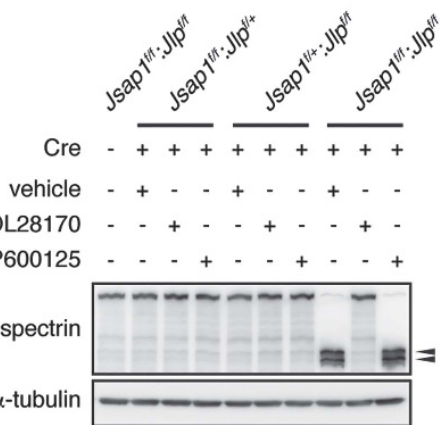

\section{f}

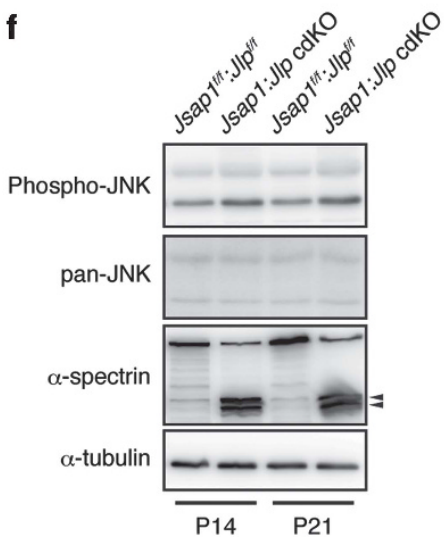

g

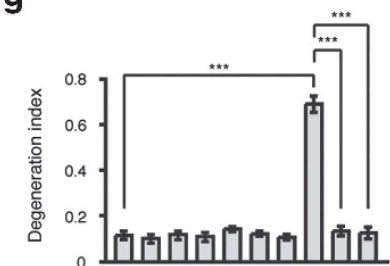

Cre - $++++++++10 \mathrm{dpi}$ vehicle - + - + + - + - -

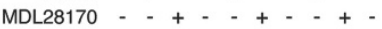

SP600125 - - + - + + - +

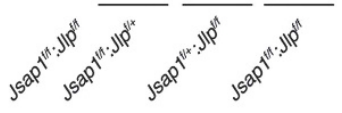

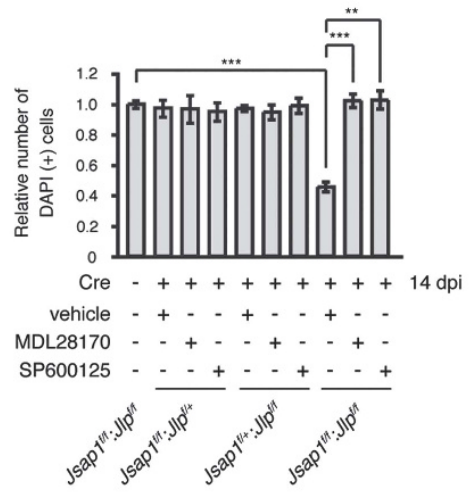


that inhibiting either JNK or calpain activity was sufficient to block axonal degeneration and neuronal loss, suggesting that JNK and calpain have cooperative roles.

In summary, we discovered that JSAP1 and JLP are essential for preventing neuronal degeneration in mice. We further identified JSAP1 and JLP as positive regulators of kinesin-1-dependent axonal transport with functional redundancy, in which the binding of JSAP1 and JLP to kinesin-1 $\mathrm{KHC}$ is crucial for kinesin-1's interactions with MTs. Finally, we suggested a molecular mechanism by which defective kinesin-1-dependent axonal transport in Jsap1:Jlp dKO neurons causes axonal degeneration and the subsequent death of the neuron.

\section{Materials and Methods}

Generation of KO mice. We constructed a targeting vector using the $10.1-\mathrm{kb}$ genomic region from $0.4-\mathrm{kb}$ downstream of exon 4 to $5.7-\mathrm{kb}$ downstream of exon 5 of the Jlp gene (Supplementary Figure S1a). The left and right arms of the targeting vector were 3.7 and $6.4 \mathrm{~kb}$, respectively. A neomycin-resistance (neo) cassette, flanked by flippase recognition target (frt) sites with a $3^{\prime}$ loxP site, was inserted between the left and right arms. An additional loxP site was inserted 0.9-kb downstream of exon 5 in the right arm. The diphtheria toxin-A (DT-A) gene was introduced for negative selection. The targeting vector was linearized with Notl, and genes were targeted in 129/Ola E14-1 ES cells as described previously. ${ }^{20}$ Homologous recombinants were identified by polymerase chain reaction (PCR) and Southern blotting (Supplementary Figure S1b). Two independent ES clones gave rise to germ-line chimeras. The mutant mice were crossed with CAG-flpe transgenic mice ${ }^{37}$ to remove the frt-flanked neo cassette. The heterozygous mice $\left(\mathrm{J} / p^{t /+}\right)$ were crossed with $\mathrm{Jsap}^{t /+}$ mice ${ }^{20}$ to generate $\mathrm{Jsap} 1^{t /+}: \mathrm{J} / \mathrm{p}^{t /+}$ mice. These mice, as well as Emx1-Cre knock-in mice, ${ }^{24}$ were backcrossed for $>10$ generations onto a C57BL/6J background and used to generate single Jsap1 cKO or Jlp cKO, and Jsap1:Jlp cdKO mice. The Emx1-Cre mice were provided by the RIKEN BRC through the National Bio-Resource Project of the MEXT, Japan. All experimental procedures involving animals were approved by the Institutional Animal Care and Use Committee of Kanazawa University.

Plasmids. The mammalian expression plasmids pCL20c-CMV-HA-JSAP1 WT and pCL20c-CMV-eGFP were described previously. ${ }^{38}$ To generate expression plasmids for the mutant JSAP1s pCL20c-CMV-HA-JSAP1-LZ, $\triangle$ KBD and LZ/ $\triangle K B D$, substitutions (L431A, L438A, L445A and L452A) at the leucine zipper domain and/or deletion of the KBD (residues 51-81) were introduced, and the respective mutant JSAP1 CDNAs were inserted into pCL20c-CMV. Coding sequences of full-length mouse JLP and a deletion mutant of KBD (residues 4575) were inserted into PCL20c-CMV to generate PCL20c-CMV-HA-JLP WT and $\triangle \mathrm{KBD}$, respectively. To generate PCL20c-CMV-DN-Capn2-HA, we subcloned the coding sequence of full-length mouse calpain 2 (Capn2), with a histidine-to-alanine substitution at residue 262 (Arthur et al. 1995) and an HA tag sequence at the $3^{\prime}$ end, into PCL20c-CMV. The coding regions of full-length mouse APP, SYP or SYT1, the Mito of human cytochrome $c$ (Cyt c) oxidase subunit $8 \mathrm{~A}$ (residues 1-29), mouse
JSAP1 JNK-binding domain (residues 201-220), DSH of mouse Dync1h1 (residues 3187-3498) and mouse DN-c-Jun lacking residues 3-122 were fused to either the $5^{\prime}$ end (the first four cDNAs) or the $3^{\prime}$ end (the remaining cDNAs) of the eGFPcoding region, and were then subcloned into PCL20c-CMV to generate the respective $\mathrm{pCL20c-CMV}$ vectors. The expression plasmid pCL20c-CMV-G-CaMP7 was generated by inserting the entire coding sequence of G-CaMP7 (Ohkura et al. 2012) into pCL20c-CMV. To generate expression plasmids for the FRET-based ATP indicator AT1.03-YEMK (Imamura et al. 2009) and its negative mutant AT1.03R122K/R126K, ${ }^{29}$ pCL20c-CMV-AT1.03-YEMK and -R122K/R126K, the respective entire coding regions were inserted into PCL20c-CMV. Substitutions and deletions in cDNAs were introduced by overlapping PCR, as described previously. ${ }^{7}$ All PCR products were verified by sequencing.

Primary cell culture and viral infection. Hippocampus tissues dissected from E15-16 mouse embryos were trypsinized and triturated as described previously. ${ }^{38}$ The dissociated hippocampal neurons were plated onto coverslips coated with poly-L-lysine $(0.5 \mathrm{mg} / \mathrm{ml}$, Sigma-Aldrich, St. Louis, MO, USA) at a density of $2.5 \times 10^{4}$ cells per $\mathrm{cm}^{2}$ in 24-well plates. The cells were grown in Neurobasal Medium (Invitrogen, Rockville, MD, USA) supplemented with $2 \%$ B-27 (Invitrogen), $2 \mathrm{mM}$ GlutaMax-I (Invitrogen), penicillin (50 units/ml) and streptomycin $(50 \mu \mathrm{g} / \mathrm{ml})$. At 2 days in vitro (DIV), cytosine $\beta$-D-arabinofuranoside $(5 \mu \mathrm{M}$, SigmaAldrich) was added to the culture medium to eliminate dividing cells. To generate Jsap1 KO, Jlp KO or Jsap1:Jlp dKO primary neurons, the corresponding primary neurons were infected with AxCANCre (a gift from I Saito) at 3 DIV. The pCL20c series of lentiviral vectors was produced as previously described. ${ }^{38}$ Primary neurons were infected with the lentiviruses 1 day before AxCANCre infection, that is, at 2 DIV, with one exception: the eGFP-DSH lentivirus infection was performed at 5 DIV, because neurons expressing eGFP-DSH developed an abnormal morphology at 13 d.p.i. independently of the Jsap1 or Jlp deletions. For the rescue experiments in Figure $5 b$ (and Supplementary Figures S5b), serial infections of HA-JSAP1 (or HAJLP) and APP-eGFP lentiviruses were performed at 1 and 2 DIV, respectively. In some experiments, bepridil (10 $\mu \mathrm{M}$, Sigma-Aldrich), MDL28170 (10 $\mu \mathrm{M}$, Millipore, Bedford, MA, USA) or SP600125 (20 $\mu$ M, Sigma-Aldrich) was dissolved in dimethyl sulfoxide and added to the culture medium (see Figure legends).

Immunoblotting. Total cell lysates were prepared and analyzed by immunoblotting as previously described ${ }^{38}$ using anti-JSAP1 (Miura et al. 2006) $(0.33 \mu \mathrm{g} / \mathrm{ml})$, anti-JLP ${ }^{21}(2 \mu \mathrm{g} / \mathrm{ml})$, anti- $\alpha$-spectrin (1:2000; MAB1622), anti-KHC (1:1000; MAB1614) from Millipore, anti-Phospho-JNK (\#9251), anti-pan-JNK (\#9258) (both diluted 1: 1000, from Cell Signaling, Boston, MA, USA), anti- $\alpha$-tubulin (1:3000; T5168), anti-Cyt c (1:5000; C5723), anti-SYP (1:500; S5768), antiSNAP-25 (1:2500; S5187) from Sigma-Aldrich, anti-COX IV (1:1000; ab14744; Abcam, Cambridge, UK), anti-SYT1 (1:1000; \#105002; Synaptic Systems, Göttingen, Germany) and anti-VAMP2 (1 : 1000; \#018-15791; Wako, Osaka, Japan) antibodies. Proteins were visualized by Immobilon Western Chemiluminescence HRP Substrate (Millipore).

Real-time PCR. Cerebral cortex total RNAs were isolated with Sepasol-RNA I Super G (Nacalai Tesque, Kyoto, Japan). Quantitative reverse-transcription PCR (qRT-PCR) was carried out as previously described, ${ }^{38}$ using the following primers: Rgs8, 5'-ATGAGGACCAGTCAACGACA-3' and 5'-TAAGGCAGCCATACAGG CAT-3'; ER81, 5'-TACCCCATGGACCACAGATT-3' and 5'-GACTGGGTCGTGGT

\footnotetext{
Figure 7 Increased intra-axonal $\mathrm{Ca}^{2+}$ causes JNK- and calpain-mediated axonal degeneration and neuronal death. (a) Increased [Ca $\left.{ }^{2+}\right] \mathrm{i}$ in Jsap1:Jlp dKO hippocampal

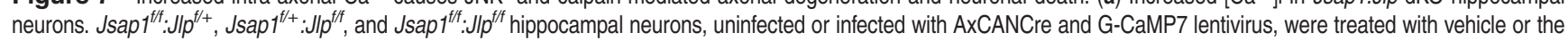
inhibitors bepridil or SP600125, then stained with anti-GFP and anti-Tau antibodies at 5 d.p.i. Bottom panels show magnified views of the boxed areas in the top panels. (b) Quantification of the results in a. The ratio of mean G-CaMP7 intensity to the mean immunofluorescence signal of eGFP in hippocampal neurons was calculated, and was quantified as the ratio of the relative G-CaMP7 intensity to the mean immunofluorescence signal of Tau. (c) JNK activation in Jsap1:Jlp dKO hippocampal neurons. Jsap $1^{t / f}$ :JIp ${ }^{f /+}$, $J \operatorname{sap} 1^{t / t}: J / p^{f / f}$, and $J s a p^{t / f}: J / p^{t / f}$ hippocampal neurons, uninfected or infected with AxCANCre, were treated with vehicle or bepridil as indicated and then stained with anti-Phospho-JNK and anti-pan-JNK antibodies at 5 d.p.i. Bottom panels show magnified views of the boxed areas in the top panels. (d) Quantification of the ratio of the mean Phospho-JNK immunofluorescence intensity to that of pan-JNK in c. (e) Calpain activation in Jsap1:Jlp dKO hippocampal neurons. AxCANCre-infected or -uninfected Jsap ${ }^{\text {ff/: }}$

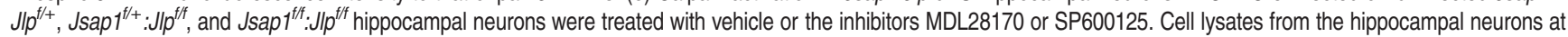
5 d.p.i. were immunoblotted with anti- $\alpha$-spectrin and anti- $\alpha$-tubulin antibodies. Arrowheads indicate the positions of $\alpha$-spectrin cleavage. (f) JNK and calpain activation in Jsap1: $\mathrm{JIp} \mathrm{cdKO} \mathrm{mice.} \mathrm{Cell} \mathrm{lysates} \mathrm{from} \mathrm{control} \mathrm{and} \mathrm{cdKO} \mathrm{mice} \mathrm{at} \mathrm{P14} \mathrm{and} \mathrm{P21} \mathrm{were} \mathrm{analyzed} \mathrm{by} \mathrm{immunoblotting} \mathrm{with} \mathrm{the} \mathrm{indicated} \mathrm{antibodies.} \mathrm{Arrowheads} \mathrm{indicate} \mathrm{the} \mathrm{positions} \mathrm{of}$ $\alpha$-spectrin cleavage. (g) MDL28170 and SP600125 suppressed axonal degeneration (left) and neuronal loss (right) in Jsap1:Jlp dKO hippocampal neurons. AxCANCre-infected

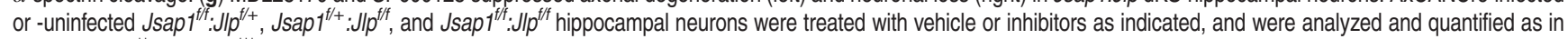
Figures $2 \mathrm{c}-$ f. ${ }^{* *} P<0.01 ;{ }^{* * *} P<0.001$; NS, not significant. Scale bars, $50 \mu \mathrm{m}$
} 
a

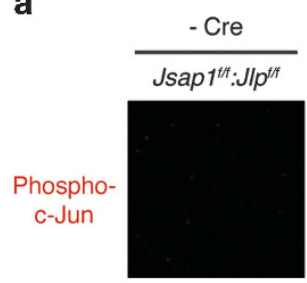

DAPI

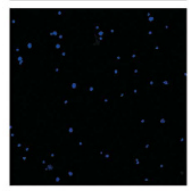

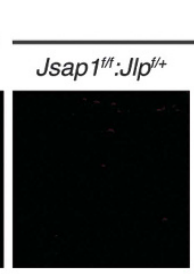

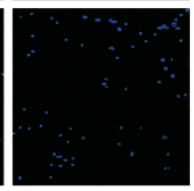

+ Cre
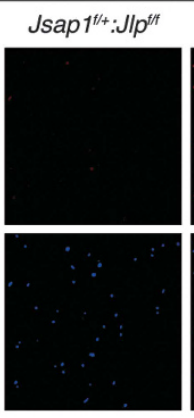
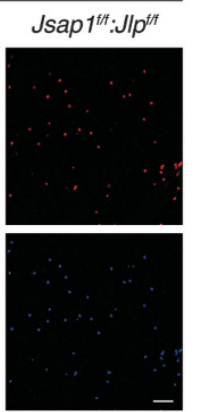

b

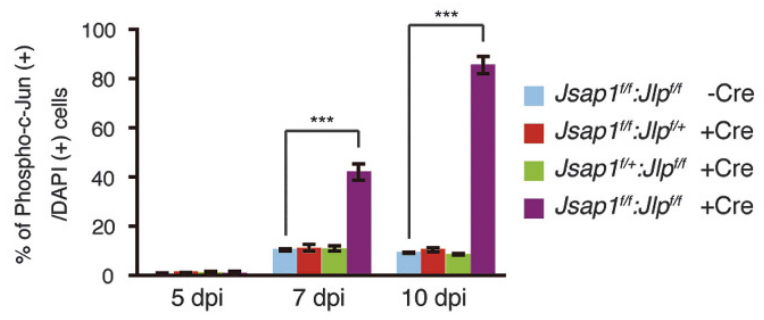

C

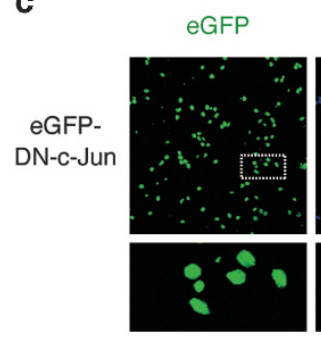

DAPI
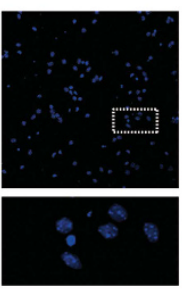

d

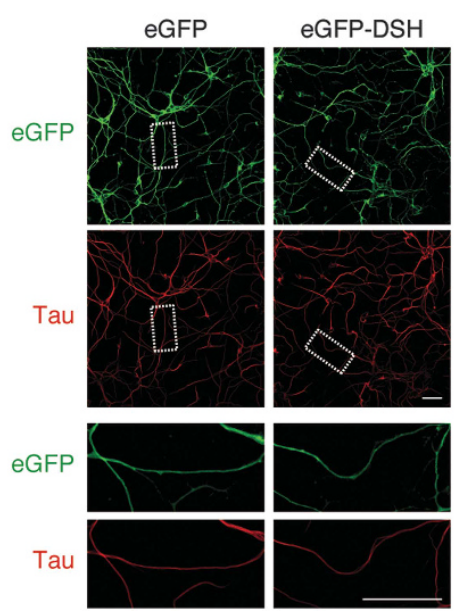

Tau

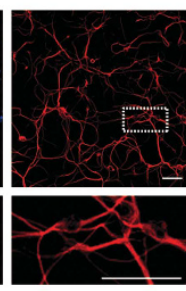

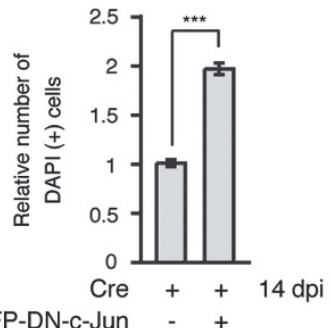

eGFP-DN-c-Jun - +

e
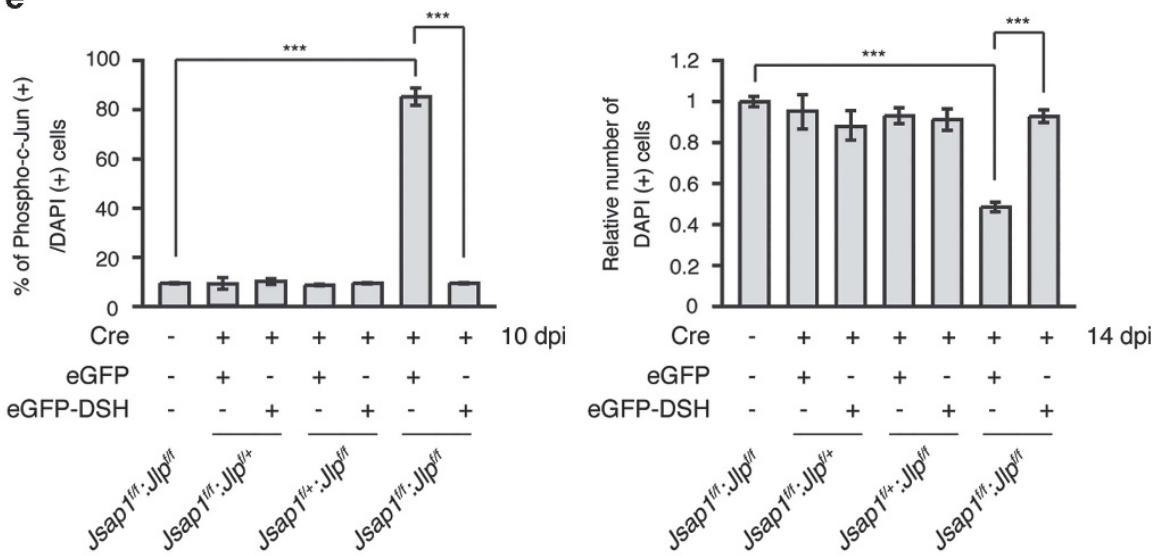

Figure 8 Retrogradely transported axonal JNK mediates neuronal death by activating c-Jun. (a) Increased numbers of Phospho-c-Jun-positive cells in cultures of Jsap1:Jlp dKO hippocampal neurons. AxCANCre-infected or -uninfected hippocampal neurons were stained with an anti-Phospho-c-Jun antibody and DAPI at 10 d.p.i., as indicated. (b) Counts of Phospho-c-Jun-positive cells, as in a, at the indicated d.p.i. (c) Neuronal loss in Jsap1:Jlp dKO cultures was suppressed by eGFP-DN-c-Jun. Lentivirally expressed eGFP-DN-c-Jun was localized exclusively to the nucleus (left). Lower panels show magnified views of the boxed areas in the upper panels. Axons were marked by Tau staining. $J$ sap $1^{t / f}: J_{1} p^{t / f}$ hippocampal neurons were uninfected or infected with eGFP-DN-c-Jun lentiviruses and AxCANCre as indicated, and the numbers of DAPI-positive cells were analyzed and quantified as in Figures $2 \mathrm{e}$ and $\mathrm{f}$ (right). (d) Lentivirally expressed eGFP and eGFP-DSH in Jsap $1^{t /+}: \mathrm{J} / \mathrm{p}^{t /+}$ hippocampal neurons. Both eGFP and eGFP-DSH were widely distributed in the neurons, with similar distribution patterns. Bottom panels show magnified views of the boxed areas in the top panels. (e) Increases in the number of

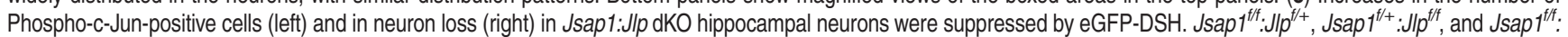
$\mathrm{J} p^{t f /}$ hippocampal neurons, uninfected or infected with AxCANCre and eGFP or eGFP-DSH lentiviruses, as indicated, were analyzed and quantified as in a and $\mathbf{b}$, and Figures $2 \mathrm{e}$ and f. ${ }^{* \star} P<0.001$. Scale bars, $50 \mu \mathrm{m}$

\section{ACTCCT-3'; and Gapdh, 5'-AAATGGTGAAGGTCGGTGTG-3' and 5'-TGAAGG GGTCGTTGATGG-3'.}

MT-binding assay. Primary cultured hippocampal neurons were homogenized in ice-cold BRB80 buffer (80 mM PIPES pH 7.0, $1 \mathrm{mM} \mathrm{MgCl}_{2}, 1 \mathrm{mM}$ EDTA and $1 \%$ TritonX-100) containing $25 \mathrm{mM} \beta$-glycerophosphate, $1 \mathrm{mM} \mathrm{Na}_{3} \mathrm{VO}_{4}, 1 \mathrm{mM} \mathrm{NaF}$ and protease inhibitor cocktail (Sigma-Aldrich), using a 30-gauge syringe. Cell lysates were centrifuged at $16000 \mathrm{~g}$ for $5 \mathrm{~min}$ at $4^{\circ} \mathrm{C}$, the supernatants were centrifuged at 54000 r.p.m. for $3 \mathrm{~min}$ in a Beckman TLA120.2 rotor at $4^{\circ} \mathrm{C}$, and $300 \mu \mathrm{g}$ total protein from the clarified supernatants was incubated with $20 \mu \mathrm{M}$ taxol (Wako),
$2.5 \mathrm{mM}$ AMP-PNP (Nacalai Tesque) and $0.1 \mathrm{mg} / \mathrm{ml}$ taxol-stabilized MTs (MT002; Cytoskeleton, Denver, CO, USA) for $30 \mathrm{~min}$ at room temperature. This mixture was loaded on top of a cushion of $20 \%$ sucrose in BRB80 buffer containing $20 \mu \mathrm{M}$ taxol, and was centrifuged for $10 \mathrm{~min}$ at 49000 r.p.m. using a Beckman TLA120.2 rotor at room temperature. The pellets were resuspended in sodium dodecyl sulfate (SDS) sample buffer $(62.5 \mathrm{mM}$ Tris- $\mathrm{HCl}$ pH $6.8,2 \%$ SDS, $10 \%$ glycerol, $5 \% 2-$ mercaptoethanol and $0.02 \%$ bromophenol blue) and analyzed by immunoblotting.

Preparation of mitochondria-enriched fractions. Primary cultured hippocampal neurons were homogenized in ice-cold mitochondrial isolation buffer 
(IB) (0.32 M sucrose, $1 \mathrm{mM}$ EDTA and $10 \mathrm{mM}$ Tris- $\mathrm{HCl} \mathrm{pH}$ 7.4). Homogenates were centrifuged at $1300 \mathrm{~g}$ for $3 \mathrm{~min}$, the supernatant was centrifuged at $21000 \mathrm{~g}$ for $10 \mathrm{~min}$ and the pellet was resuspended in 15\% Percoll (GE Healthcare, Buckinghamshire, UK). This suspension was overlaid on a Percoll gradient containing 23\% Percoll layered above $40 \%$ Percoll, and the gradient was centrifuged at $30000 \mathrm{~g}$ for $5 \mathrm{~min}$. The mitochondrial fraction was harvested from the interface of the 23 and $40 \%$ layers of Percoll, was supplemented to a final concentration of $1.4 \mathrm{mg} / \mathrm{ml}$ bovine serum albumin in IB and was centrifuged at $16000 \mathrm{~g}$ for $10 \mathrm{~min}$. The pellet was resuspended in RIPA buffer $(50 \mathrm{mM}$ Tris-HCl $\mathrm{pH} 7.4,150 \mathrm{mM} \mathrm{NaCl}, 1 \%$ Nonidet P-40, $1 \%$ sodium deoxycholate, $0.1 \%$ SDS and $1 \mathrm{mM} \mathrm{EDTA}$ ) and subjected to immunoblotting. All centrifugation procedures were carried out at $4^{\circ} \mathrm{C}$.

Histological analysis and immunofluorescence. Mice were deeply anesthetized and fixed by transcardial perfusion with $4 \%$ paraformaldehyde (PFA); brain samples were postfixed as described previously. ${ }^{38}$ The samples were dehydrated or cryoprotected in 30\% sucrose and embedded in paraffin or TissueTek OCT compound (Sakura Finetek, Kyoto, Japan). For Nissl staining, 10- $\mu \mathrm{m}$-thick paraffin sections were stained with cresyl violet. Immunohistochemistry was performed as previously described. ${ }^{38}$ The TUNEL assay was performed on $25-\mu \mathrm{m}$ thick frozen sections using the In Situ Cell Death Detection Kit TMR Red (Roche, Basel, Switzerland), according to the manufacturer's protocol. Cultured hippocampal neurons were fixed with $4 \%$ PFA for $30 \mathrm{~min}$ at room temperature, and were analyzed by immunocytochemistry as previously described. ${ }^{41}$ The following primary antibodies were used: anti-JSAP1 (Miura et al. 2006) $(1 \mu \mathrm{g} / \mathrm{ml})$, anti-JLP ${ }^{21}$ $(2 \mu \mathrm{g} / \mathrm{ml})$, anti-Phospho-JNK (\#9251), anti- Phospho-c-Jun (\#9261) (both diluted $1: 100)$, anti-active caspase-3 $(1: 200$, \#9661) from Cell Signaling, anti-Tau (1:500; AHB0042; Invitrogen or 1: 500; A0024; Dako, Glostrup, Denmark), anti-HA (1:300; \#1867423; Roche), anti-GFP (1:300; AB3080), anti-APP (1:50; MAB348) from Millipore, anti-pan-JNK ( 1 : 100; \#610628; BD Biosciences, San Jose, CA, USA), anti-GFAP (1:500; G3893), anti-SYP (1:200; S5768) from Sigma-Aldrich, antiSYT1 (1 : 100; \#105002; Synaptic Systems) and anti-VAMP2 (1: 300; \#018-15791; Wako). We used the following secondary antibodies: Alexa 350-, 488- and 568conjugated anti-mouse IgG, and Alexa 350-, 488- and 568-conjugated anti-rabbit IgG (all diluted at 1:1000; Invitrogen) antibodies. In some experiments, nuclei were stained with 4,6-diamidino-2-phenylindole (DAPI) (Sigma-Aldrich), and mitochondria in living cells were detected with MitoTracker Orange $\mathrm{CM}_{2} \mathrm{H}_{2}$ TMRos (Invitrogen).

Image acquisition and quantitative analysis. Unless otherwise noted images were captured by a confocal laser scanning microscope (LSM510 META, Carl Zeiss, Oberkochen, Germany) using a $20 \times$ or $40 \times$ objective lens, with or without $2 \times$ digital zoom, below saturation at a resolution of $512 \times 512$ pixels ( 8 bit). All the images were acquired without Z-stacking. For quantitative analysis of cultured neurons, confocal images of five randomly chosen fields per coverslip were acquired with a $20 \times$ objective lens. To quantify the G-CaMP7 and Phospho-JNK fluorescence levels, the fluorescence intensity of each entire field was measured using Image ( $\mathrm{NIH}$, Bethesda, MD, USA) and quantified as described in the corresponding Figure legend. Cells positive for active caspase-3, DAPI or Phosphoc-Jun were counted in the respective image fields. For quantitative analysis of swollen axons, confocal images of 10 randomly chosen axons were acquired using a $40 \times$ objective lens with $2 x$ digital zoom. To quantify the fluorescence levels of MitoTracker, APP, SYP, SYT1 and Tau, the fluorescence intensity of each swollen axon was measured using Image $\mathrm{J}$ and was quantified as described in Figure 3 (legend). To acquire FRET images, cyan fluorescent protein (CFP, FRET-donor) was excited at $405 \mathrm{~nm}$ with a diode laser $(4.5 \mathrm{~mW})$. The CFP and yellow fluorescent protein (YFP, FRET-acceptor) emissions were collected through 470-500-nm and $530-600-\mathrm{nm}$ band pass filters, respectively. For the direct excitation of YFP, the 514$\mathrm{nm}$ laser line of an argon laser $(0.75 \mathrm{~mW})$ was used, and the emission was collected through a 530-600-nm band pass filter. Images were acquired from 10 randomly selected axons per experimental condition with a $40 \times$ objective lens with or without a $4 \times$ zoom. The images were captured at a portion of the axon $150 \mu \mathrm{m}$ away from the soma and $100 \mu \mathrm{m}$ in length. Fluorescence intensities in the CFP, FRET and YFP channels were measured using ImageJ, and quantified as described in Supplementary Figure S6 (legend). Axonal degeneration was quantified as described by Sasaki et al. ${ }^{42}$ Briefly, fluorescence images of Tau-stained axons were binarized to convert all pixels corresponding to axons to black, and all other regions to white. The total axonal area was determined by the total number of black pixels. Non-degenerated axons appeared as continuous tracts, whereas degenerated axons appeared as fragments, beads or particles. Degenerated axons were detected using the particle analyzer in ImageJ. A degeneration index was calculated as the ratio of the area of degenerated axon to the total axon area. Quantitative data were expressed as the mean \pm S.E.M. of three independent experiments.

For time-lapse imaging, primary hippocampal neurons were cultured as described above, using 35-mm glass-bottom dishes (Iwaki, Chiba, Japan) instead of 24-well plates. Cells were incubated at $37^{\circ} \mathrm{C}$ in $5 \% \mathrm{CO}_{2}$ during imaging (CZl-3; Carl Zeiss). Axonal processes were chosen for recording based on known morphological characteristics. Axons were identified as processes stemming from the soma that were two to three times longer than other processes. Time-lapse images were acquired with a $63 \times$ water-immersion lens and a $2 \times$ zoom at a resolution of $512 \times 256$ pixels ( 8 bit), using $488 \mathrm{~nm}(0.25 \mathrm{~mW})$ and $543 \mathrm{~nm}(0.5 \mathrm{~mW})$ excitation for eGFP and MitoTracker Orange, respectively. Each series of images was recorded for five randomly selected axons per experimental condition. A portion of the axon that was at least $100 \mu \mathrm{m}$ away from the soma was recorded for 5 -s (for Mito-eGFP, MitoTracker and APP-eGFP) or 60-s (for SYP-eGFP and SYT1-eGFP) intervals, for a total of 60 frames. To analyze the movement of fluorescence-tagged mitochondria, APP, SYP or SYT1, kymographs were generated from time-lapse videos using the Kymograph plugin for ImageJ. Mitochondria, APP, SYP and SYT1 were defined as mobile if there was a displacement of at least $5 \mu \mathrm{m}$. Stationary and motile mitochondria, APP, SYP and SYT1 were counted, and quantitative data were expressed as the mean \pm S.E.M. of three independent experiments.

Statistical analysis. Kaplan-Meier curves and the relative intensities of motor cargoes in swollen axons were analyzed by log-rank test and one-way ANOVA, respectively. All other statistical significance was determined by a two-tailed unpaired Student's $t$-test. Values of $P<0.05$ were considered to be statistically significant.

\section{Conflict of Interest}

The authors declare no conflict of interest.

Acknowledgements. We are grateful to I Saito (University of Tokyo) and $\mathrm{H}$ Imamura (Kyoto University) for providing the AxCANCre and the AT1.03 constructs, respectively, and to $\mathrm{H}$ Tanaka (Ritsumeikan University) and T Yoshihara (Kanazawa University) for helpful discussions. This work was supported in part by Grants-in-Aid from the Ministry of Education, Culture, Sports, Science, and Technology of Japan.

1. Perlson E, Maday S, Fu MM, Moughamian AJ, Holzbaur EL. Retrograde axonal transport: pathways to cell death? Trends Neurosci 2010; 33: 335-344.

2. Millecamps S, Julien JP. Axonal transport deficits and neurodegenerative diseases. Nat Rev Neurosci 2013; 14: 161-176.

3. Verhey KJ, Hammond J. Traffic control: regulation of kinesin motors. Nat Rev Mol Cell Biol 2009; 10: 765-777

4. Hirokawa N, Noda Y, Tanaka Y, Niwa S. Kinesin superfamily motor proteins and intracellular transport. Nat Rev Mol Cell Biol 2009; 10: 682-696.

5. Whitmarsh AJ, Cavanagh J, Tournier C, Yasuda J, Davis RJ. A mammalian scaffold complex that selectively mediates MAP kinase activation. Science 1998; 281: 1671-1674.

6. Yasuda J, Whitmarsh AJ, Cavanagh J, Sharma M, Davis RJ. The JIP group of mitogenactivated protein kinase scaffold proteins. Mol Cell Biol 1999; 19: 7245-7254.

7. Ito M, Yoshioka K, Akechi M, Yamashita S, Takamatsu N, Sugiyama K et al. JSAP1, a novel Jun N-terminal protein kinase (JNK) that functions as scaffold factor in the JNK signaling pathway. Mol Cell Biol 1999; 19: 7539-7548.

8. Kelkar N, Gupta S, Dickens M, Davis RJ. Interaction of a mitogen-activated protein kinase signaling module with the neuronal protein JIP3. Mol Cell Biol 2000; 20: 1030-1043

9. Lee CM, Onésime D, Reddy CD, Dhanasekaran N, Reddy EP. JLP: A scaffolding protein that tethers JNK/p38MAPK signaling modules and transcription factors. Proc Natl Acad Sci USA 2002; 99: 14189-14194.

10. Kelkar N, Standen CL, Davis RJ. Role of the JIP4 scaffold protein in the regulation of mitogen-activated protein kinase signaling pathways. Mol Cell Biol 2005; 25: 2733-2743.

11. Jagadish N, Rana R, Selvi R, Mishra D, Garg M, Yadav S et al. Characterization of a novel human sperm-associated antigen 9 (SPAG9) having structural homology with c-Jun $\mathrm{N}$-terminal kinase-interacting protein. Biochem J 2005; 389: 73-82.

12. Verhey KJ, Meyer D, Deehan R, Blenis J, Schnapp BJ, Rapoport TA et al. Cargo of kinesin identified as JIP scaffolding proteins and associated signaling molecules. J Cell Biol 2001; 152: 959-970.

13. Nguyen Q, Lee CM, Le A, Reddy EP. JLP associates with kinesin light chain 1 through a novel leucine zipper-like domain. J Biol Chem 2005; 280: 30185-30191. 
14. Horiuchi D, Barkus RV, Pilling AD, Gassman A, Saxton WM. APLIP1, a kinesin binding JIP-1/JNK scaffold protein, influences the axonal transport of both vesicles and mitochondria in Drosophila. Curr Biol 2005; 15: 2137-2141.

15. Bowman AB, Kamal A, Ritchings BW, Philp AV, McGrail M, Gindhart JG et al. Kinesindependent axonal transport is mediated by the Sunday driver (SYD) protein. Cell 2000; 103: 583-594.

16. Byrd DT, Kawasaki M, Walcoff M, Hisamoto N, Matsumoto K, Jin Y. UNC-16, a JNKsignaling scaffold protein, regulates vesicle transport in C. elegans. Neuron 2001; 32 : 787-800.

17. Kennedy NJ, Martin G, Ehrhardt AG, Cavanagh-Kyros J, Kuan CY, Rakic P et al. Requirement of JIP scaffold proteins for NMDA-mediated signal transduction. Genes Dev 2007; 21: 2336-2346.

18. Kelkar N, Delmotte MH, Weston CR, Barrett T, Sheppard BJ, Flavell RA et al. Morphogenesis of the telencephalic commissure requires scaffold protein JNK-interacting protein 3 (JIP3). Proc Natl Acad Sci USA 2003; 100: 9843-9848.

19. Ha HY, Cho IH, Lee KW, Lee KW, Song JY, Kim KS et al. The axon guidance defect of the telencephalic commissures of the JSAP1-deficient brain was partially rescued by the transgenic expression of JIP1. Dev Biol 2005; 277: 184-199.

20. Iwanaga A, Sato T, Sugihara K, Hirao A, Takakura N, Okamoto $H$ et al. Neural-specific ablation of the scaffold protein JSAP1 in mice causes neonatal death. Neurosci Lett 2007; 429: 43-48.

21. Iwanaga A, Wang G, Gantulga D, Sato T, Baljinnyam T, Shimizu $K$ et al. Ablation of the scaffold protein JLP causes reduced fertility in male mice. Transgenic Res 2008; 17: 1045-1058.

22. Huang SH, Duan S, Sun T, Wang J, Zhao L, Geng Z, Yan J, Sun HJ, Chen ZY. JIP3 mediates TrkB axonal anterograde transport and enhances BDNF signaling by directly bridging TrkB with kinesin-1. J Neurosci 2011; 31: 10602-10614.

23. Deng CY, Lei WL, Xu XH, Ju XC, Liu Y, Luo ZG. JIP1 mediates anterograde transport of Rab10 cargos during neuronal polarization. J Neurosci 2014; 34: 1710-1723.

24. Iwasato T, Nomura R, Ando R, Ikeda T, Tanaka M, Itohara S. Dorsal telencephalon-specific expression of Cre recombinase in PAC transgenic mice. Genesis 2004; 38: 130-138.

25. Kanegae $Y$, Lee G, Sato $Y$, Tanaka M, Nakai M, Sakaki T et al. Efficient gene activation in mammalian cells by using recombinant adenovirus expressing site-specific Cre recombinase. Nucleic Acids Res 1995; 23: 3816-3821.

26. Sun F, Zhu C, Dixit R, Cavalli V. Sunday Driver/JIP3 binds kinesin heavy chain directly and enhances its motility. EMBO J 2011; 30: 3416-3429.

27. Wang $X$, Schwarz TL. The mechanism of $\mathrm{Ca}^{2+}$-dependent regulation of kinesin-mediated mitochondrial motility. Cell 2009; 136: 163-174.
28. Stirling DP, Stys PK. Mechanisms of axonal injury: internodal nanocomplexes and calcium deregulation. Trends Mol Med 2010; 16: 160-170.

29. Imamura $H$, Nhat KP, Togawa $H$, Saito $K$, lino $R$, Kato-Yamada $Y$, Nagai $T$, Noji $H$. Visualization of ATP levels inside single living cells with fluorescence resonance energy transfer-based genetically encoded indicators. Proc Natl Acad Sci USA 2009; 106: 15651-15656.

30. Ohkura M, Sasaki T, Sadakari J, Gengyo-Ando K, Kagawa-Nagamura Y, Kobayashi C et al. Genetically encoded green fluorescent $\mathrm{Ca} 2+$ indicators with improved detectability for neuronal $\mathrm{Ca}^{2+}$ signals. PLoS One 2012; 7: e51286.

31. Vosler PS, Brennan CS, Chen J. Calpain-mediated signaling mechanisms in neuronal injury and neurodegeneration. Mol Neurobiol 2008; 38: 78-100.

32. Davis RJ. Signal transduction by the JNK group of MAP kinases. Cell 2000; 103: 239-252.

33. Kamal A, Stokin GB, Yang Z, Xia CH, Goldstein LS. Axonal transport of amyloid precursor protein is mediated by direct binding to the kinesin light chain subunit of kinesin-l. Neuron 2000; 28: 449-459.

34. Glater EE, Megeath LJ, Stowers RS, Schwarz TL. Axonal transport of mitochondria requires milton to recruit kinesin heavy chain and is light chain independent. J Cell Biol 2006; 173: $545-557$.

35. Morrison DK, Davis RJ. Regulation of MAP kinase signaling modules by scaffold proteins in mammals. Annu Rev Cell Dev Biol 2003; 19: 91-118.

36. Dhanasekaran DN, Kashef K, Lee CM, Xu H, Reddy EP. Scaffold proteins of MAP-kinase modules. Oncogene 2007; 26: 3185-3202.

37. Kanki $H$, Suzuki $H$, Itohara S. High-efficiency CAG-FLPe deleter mice in C57BL/6J background. Exp Anim 2006; 55: 137-141.

38. Sato T, Torashima T, Sugihara K, Hirai H, Asano M, Yoshioka K. The scaffold protein JSAP1 regulates proliferation and differentiation of cerebellar granule cell precursors by modulating JNK signaling. Mol Cell Neurosci 2008; 39: 569-578.

39. Arthur JS, Gauthier S, Elce JS. Active site residues in m-calpain: identification by site-directed mutagenesis. FEBS Lett 1995; 368: 397-400.

40. Miura E, Fukaya M, Sato T, Sugihara K, Asano M, Yoshioka $\mathrm{K}$ et al. Expression and distribution of JNK/SAPK-associated scaffold protein JSAP1 in developing and adult mouse brain. J Neurochem 2006; 97: 1431-1446.

41. Sato T, Enkhbat A, Yoshioka K. Role of plasma membrane localization of the scaffold protein JSAP1 during differentiation of cerebellar granule cell precursors. Genes Cells 2011; 16: 58-68.

42. Sasaki Y, Vohra BP, Lund FE, Milbrandt J. Nicotinamide mononucleotide adenylyl transferase-mediated axonal protection requires enzymatic activity but not increased levels of neuronal nicotinamide adenine dinucleotide. J Neurosci 2009; 29: 5525-5535.

Supplementary Information accompanies this paper on Cell Death and Differentiation website (http://www.nature.com/cdd) 